\title{
A Survey of Vapors in the Headspaces of Single-Shell Waste Tanks
}

L. M. Stock ${ }^{(\mathrm{a})}$

J. L. Huckaby

October 2000

Prepared for the U.S. Department of Energy under Contract DE-AC06-76RLO 1830

Pacific Northwest National Laboratory Richland, Washington 99352

(a) Private consultant, Portland, Oregon 



\section{DISCLAIMER}

This report was prepared as an account of work sponsored by an agency of the United States Government. Neither the United States Government nor any agency thereof, nor any of their employees, make any warranty, express or implied, or assumes any legal liability or responsibility for the accuracy, completeness, or usefulness of any information, apparatus, product, or process disclosed, or represents that its use would not infringe privately owned rights. Reference herein to any specific commercial product, process, or service by trade name, trademark, manufacturer, or otherwise does not necessarily constitute or imply its endorsement, recommendation, or favoring by the United States Government or any agency thereof. The views and opinions of authors expressed herein do not necessarily state or reflect those of the United States Government or any agency thereof. 


\section{DISCLAIMER}

Portions of this document may be illegible in electronic image products. Images are produced from the best available original document. 


\section{Contents}

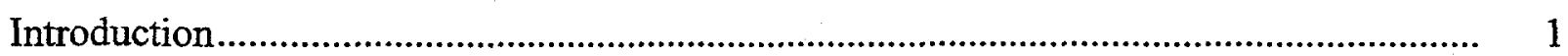

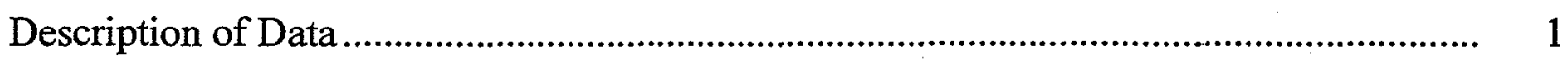

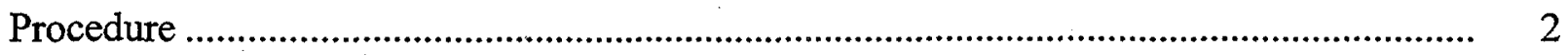

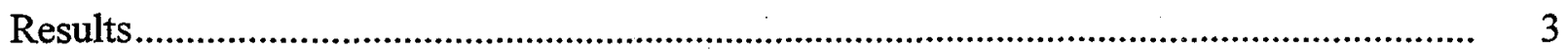

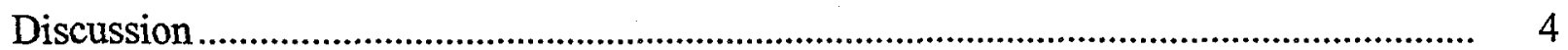

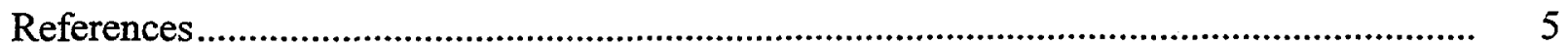




\section{Tables}

1a Alkanes

1b Alkanes Observed in One Tank at Concentration Less Than $0.025 \mathrm{mg} / \mathrm{m}^{3} \ldots \ldots \ldots \ldots . . . . . .9$

2a Cycloalkanes .......................................................................................... 10

2b Cycloalkanes Observed in One Tank at Concentration Less Than $0.025 \mathrm{mg} / \mathrm{m}^{3} \ldots \ldots . . \quad 12$

3a Alkenes and Alkadienes ............................................................................. 13

3b Alkenes and Alkadienes Observed in One Tank at Concentration Less Than $0.025 \mathrm{mg} / \mathrm{m}^{3}$

4a Alkynes.

$4 \mathrm{~b} \quad$ Alkynes Observed in One Tank at Concentration Less Than $0.025 \mathrm{mg} / \mathrm{m}^{3}$......

5a Benzene, Biphenyl, Napthalene, Fluorene and Their Alkyl and Alkenyl

Derivatives.

5b Benzene, Biphenyl, Napthalene, Fluorene and Their Alkyl and Alkenyl Derivatives Observed in One Tank at Concentration Less Than $0.025 \mathrm{mg} / \mathrm{m}^{3}$ 20

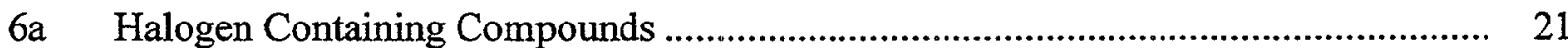

6b Halogen Containing Compounds Observed in One Tank at Concentration Less Than $0.025 \mathrm{mg} / \mathrm{m}^{3}$

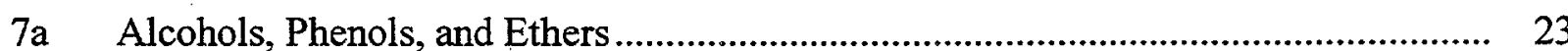

$7 \mathrm{~b}$ Alcohols, Phenols, and Ethers Observed in One Tank at Concentration Less Than $0.025 \mathrm{mg} / \mathrm{m}^{3}$

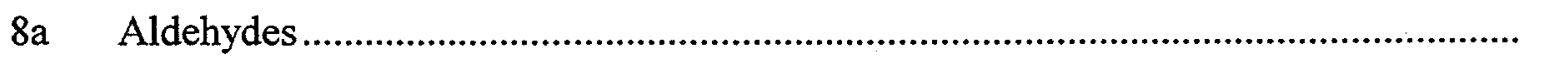

8b Aldehydes Observed in One Tank at Concentration Less Than $0.025 \mathrm{mg} / \mathrm{m}^{3} \ldots \ldots \ldots \ldots .28$ 
$9 \mathrm{~b}$ Ketones Observed in One Tank at Concentration Less Than $0.025 \mathrm{mg} / \mathrm{m}^{3} \ldots$

10a Acids

$10 \mathrm{~b}$ Acids Observed in One Tank at Concentration Less Than $0.025 \mathrm{mg} / \mathrm{m}^{3}$

11a Esters

11b Esters Observed in One Tank at Concentration Less Than $0.025 \mathrm{mg} / \mathrm{m}^{3}$

12a Nitriles 36

$12 \mathrm{~b}$ Nitriles Observed in One Tank at Concentration Less Than $0.025 \mathrm{mg} / \mathrm{m}^{3}$ 37

13a Amines and Amides 38

13b Amines and Amides Observed in One Tank at Concentration Less Than $0.025 \mathrm{mg} / \mathrm{m}^{3}$

14a Nitrous and Nitric Acid Esters, Nitrous and Nitro Compounds 40

14b Nitrous and Nitric Acid Esters, Nitrous and Nitro Compounds Observed in One Tank at Concentration Less Than $0.025 \mathrm{mg} / \mathrm{m}^{3}$

15a Heterocycles

15b Heterocycles Observed in One Tank at Concentration Less Than $0.025 \mathrm{mg} / \mathrm{m}^{3}$

16a Sulfur Compounds

16b Sulfur Compounds Observed in One Tank at Concentration Less Than $0.025 \mathrm{mg} / \mathrm{m}^{3}$

17a Silicon Compounds

17b Silicon Compounds Observed in One Tank at Concentration Less Than $0.025 \mathrm{mg} / \mathrm{m}^{3}$ 
18a Other Organic Compounds.

18b Other Organic Compounds Observed in One Tank at Concentration Less Than $0.025 \mathrm{mg} / \mathrm{m}^{3}$

19 Inorganic Compounds

20 Tanks With Highest Organic Carbon Content in Dome Space Determined by Total Non-Methane Hydrocarbon Content Measurements, GC-MS Analysis of Summa Canisters or TST Samples

21 Summary of Observations. 52 


\section{Introduction}

This report summarizes data on the organic vapors in the single-shell, high-level radioactive waste tanks at the Hanford Site to support a forthcoming toxicological study. All data were obtained from the Tank Characterization Database (TCD) (PNNL 1999). The TCD contains virtually all the available tank headspace characterization data from 1992 to the present, and includes data for 109 different single-shell waste tanks. Each single-shell tank farm and all major waste types are represented. Descriptions of the sampling and analysis methods have been given elsewhere (Huckaby et al. 1995, Huckaby et al. 1996), and references for specific data are available in the TCD. This is a revision of a report issued on March 1, 2000 (Stock and Huckaby 2000).

\section{Description of Data}

The extent to which different tank headspaces have been characterized varies greatly. The majority of tanks have been sampled only once and generally a single laboratory analyzed the samples, but some tanks have been sampled repeatedly and multiple laboratories have conducted analyses. Two types of sampling devices, triple sorbent traps (TSTs) and Summa ${ }^{\mathrm{TM}}$ canisters, were employed to ensure collection and recovery of organic compounds with a broad range of volatilities, and to provide a comparison of overlapping results.

Analyses to identify and quantify specific organic compounds have been performed on 104 of the 109 total tanks sampled. ${ }^{1}$ Samples from each of these 104 tanks were analyzed using gas chromatograph/ mass spectrometer (GC/MS) systems, with robust methods that were designed to maximize the number and types of species that could be detected. This summary report includes GC/MS results from about 1,200 tank headspace samples.

Sampling and analysis plans specified that samples be analyzed for as many as 65 organic target compounds. The target compounds were to be positively identified (i.e., GC retention time and mass spectra were matched to that of a known standard) and quantitatively measured (i.e., a multi-point calibration of the GC/MS was performed with known standards). Concentrations of target compounds were generally required to be accurate to within $30 \%$. Non-target organic compounds were tentatively identified by comparing their observed mass spectra with those in the NIST/Wiley mass spectral library, and applying both automatic search methods and professional judgment to identify the best match. Experience indicates this method for identification of organic compounds is fairly reliable for many compounds. Confidence that any given tentatively identified compound has been properly identified tends to go down as its concentration goes down, and as the number of possible chemical isomers goes up.

Concentrations of tentatively identified compounds were estimated by comparing their instrument response to that of chromatographically adjacent internal standards, and generally should be only considered to be accurate to a factor of 2 .

\footnotetext{
${ }^{1}$ In 1995 , it was decided that organic vapor speciation would not be conducted on certain tanks if their total organic vapor concentration was below $5 \mathrm{mg} / \mathrm{m}^{3}$.
} 
In addition to uncertainties in the sampling and analysis methodology, changes in the ventilation rates of the single-shell tanks directly affect the headspace concentrations of all species. If a given tank were sampled during a period when its ventilation rate was low, the measured concentrations would be higher than if the tank were sampled during a period of high ventilation rate. Ventilation rates of both the passively and actively ventilated tanks may vary by a factor of about 3 due to vent-and-balance adjustments and meteorological conditions, ${ }^{2}$ and the reported concentrations should be assumed to vary accordingly.

The TCD contains about 35,000 results for compounds detected by GC/MS organic vapor analyses. This report summarizes the results for about 1,200 compounds, of which about 500 were found in only one type of sample from a single tank. About 430 of the 1,200 compounds have maximum reported concentrations of less than $0.025 \mathrm{mg} / \mathrm{m}^{3}$. Suspect results (flagged by the analyst as suspect or associated with blank contamination) and results for ambiguously identified species (e.g., species identified as "unknown", "unknown C-12 alkanone", etc.) were not included in this report.

\section{Procedure}

Results for organic compounds have been divided into 18 tables, according to chemical classification. Individual tables are given for the alkanes; cycloalkanes; alkenes and alkadienes; alkynes; arenes; halogen compounds; alcohols, phenols, and ethers; aldehydes; ketones; acids; esters; nitriles; amines and amides; nitroso and nitro compounds; heterocycles; sulfur-containing compounds; silicon compounds; and miscellaneous organic compounds. This categorization was accomplished readily because there are very few compounds with more than one functional group. In those few cases where a compound could be included in more than one table, the compounds were included in the table of perceived greater toxicological interest (in only one table). For example, the haloalkene chloroethene was included in the halogen-containing compound table (Table 6a) and not in the alkene table (Table 3a).

Because these tables were prepared primarily for the assessment of toxicological hazards, each table is divided into two parts based on whether the highest reported concentration of the compound is above $0.025 \mathrm{mg} / \mathrm{m}^{3}$ (about $5 \mathrm{ppbv}$ ). Tables 1a, 2a, etc., contain those compounds that have reported maximum concentrations at or above $0.025 \mathrm{mg} / \mathrm{m}^{3}$, and Tables $1 \mathrm{~b}, 2 \mathrm{~b}$, etc., contain those compounds that have reported maximum concentrations below $0.025 \mathrm{mg} / \mathrm{m}^{3}$. The $0.025 \mathrm{mg} / \mathrm{m}^{3}$ threshold value was recommended by Bartley (1999) as appropriately conservative in their evaluation of the toxicological hazards associated with the organic vapors emanating from tank $\mathrm{C}-106$ during sluicing operations.

Table 19 lists results for inorganic compounds. The tanks having the highest total organic vapor concentrations are listed in Table 20 with the reported values. Summary statistics are given in Table 21.

\footnotetext{
${ }^{2}$ Single shell tanks C-104, C-105, C-106, SX-101, SX-102, SX-103, SX-104, SX-105, SX-106, SX-107, SX-108, SX-109, SX-110, SX-111, SX-113, and SX-115 are actively ventilated. Passive ventilation rates are discussed by Huckaby et al. 1998.
} 
In some instances two or more compounds coelute chromatographically. The identification of coeluting compounds presents a significant analytical challenge, and these observations were treated in the following manner. Insofar as possible, these observations were included. For example, after the numerous entries for decane in the database, there appears the entry "decane and others" at $0.036 \mathrm{mg} / \mathrm{m}^{3}$. Results of this kind were counted as an observation for the identified compound, i.e., decane. As another example, after the numerous entries for dodecane in the database, there appears the entry "dodecane and 2'-hydroxy-5'-methoxyacetophenone" at $0.095 \mathrm{mg} / \mathrm{m}^{3}$. This result was included as an observation for dodecane, but the observation for the acetophenone was not included, since it had not been observed independently.

\section{Results}

The observations for the alkanes, cycloalkanes, alkenes and alkadienes, alkynes, arenes, halogenated compounds, alcohols, phenols and ethers, aldehydes, ketones, acids, esters, amines and amides, nitriles, nitroso and nitro compounds, heterocycles, silicon compounds, sulfur compounds, and miscellaneous organic compounds appear in Tables $1 \mathrm{a}$ to $18 \mathrm{a}$, and $1 \mathrm{~b}$ to $18 \mathrm{~b}$. Table 19 lists the inorganic compounds.

The compounds are assembled in the tables beginning with the smallest molecular weight. The next columns show the total number of analytical observations for the compound, the maximum observed concentration in $\mathrm{mg} / \mathrm{m}^{3}$, the molecular weight, the maximum observed concentration in ppbv, and the tank from which the sample having the maximum concentration was obtained. The sums of the total number of observations and the maximum concentrations appear at the bottom of each table.

Supplementary information about the concentrations of the organic compounds in the tank headspaces is provided in Table 20. This table, which is also derived from information in the TCD, lists the 10 tanks with the highest total concentrations of organic carbon based on three different analytical measurements. The total non-methane hydrocarbon (TNMHC) measurement was conducted by analysis of Summa canister samples using the U.S. Environmental Protection Agency (EPA) Task Order 12 (TO-12) method. Summations of the reported concentrations by GC/MS analysis of Summa and TST samples are also given.

Finally, Table 21 provides a summary of the results. This table provides perspective on the relationship between the total number of different compounds listed in the category with the number of compounds in the same category that have been observed only once or more than once at a concentration less than $0.025 \mathrm{mg} / \mathrm{m}^{3}$. The table lists the sum of the maximum concentration of the compounds in the category in ppbv. Table 21 also lists the number of compounds reported to have concentrations in excess of 100,500 , and $1,000 \mathrm{ppbv}$ for each structural group. 


\section{Discussion}

Organic compounds are ubiquitous in the headspaces of the waste tanks. The alkanes (about 200 compounds), cycloalkanes (about 150 compounds), and alkenes (about 150 compounds) are only modestly more numerous than the other compounds with organic functional groups. There are about 120 ketones, about 110 alcohols, phenols and ethers and about 100 heterocycles (some of the heterocycles, for example the tetrahydrofurans, could be equally well listed as ethers).

The sum of the maximum concentrations of the 1,200 organic compounds is about $630 \mathrm{ppmv}$. The alkanes contribute about $250 \mathrm{ppmv}$, the alcohols and ethers contribute about $120 \mathrm{ppmv}$ (not including the heterocyclic ethers), and the ketones contribute about $60 \mathrm{ppmv}$. The sum of the maximum concentrations of the halogen compounds is also significant at about $40 \mathrm{ppmv}$.

Many semivolatile alkanes and cycloalkanes are recognized as constituents of the normal paraffinic hydrocarbon (NPH) diluent used in various Hanford extraction processes. Virtually all of the other alkanes, alkenes, alkynes, arenes, alcohols and ethers, aldehydes, ketones, acids, esters, amides and amines, nitroso and nitro compounds and nitriles can be accounted for as products of the thermally and radiolytically induced free radical and ionic fragmentation and oxidation reactions of NPH, phosphate esters, and complexants. Most of the heterocycles and miscellaneous compounds can also be accounted for in the same manner. The technical bases for these conclusions are set forth in part in Appendix L of the Organic Solvent Topical Report (Cowley et al. 1998) and in The Chemistry of Flammable Gas Generation (Stock 2000).

Few of the molecules containing halogen, silicon, or sulfur atoms can arise from the paraffinic hydrocarbon, phosphate esters or complexants. Some of the analytical observations may be spurious. However, many of the compounds that cannot be directly related to the principal source term arise from other identifiable substances. The bromine- and chlorine-containing compounds presumably arise from tetrabromoethane and the chlorocarbon solvents that were used in operations before 1980 (Klem 1990, Gerber et al. 1992). In addition, the unusual trifluoro ketone listed in Table 6 can be traced to a substance listed by Klem. However, the origins of the freons have not been definitely established. These substances apparently arise from sources other than the liquid and solid wastes. The principal silicon-containing compounds, the bis(trimethylsilyl) derivative of 2-hydroxybenzoic acid and cyclic hexa- and octamethyl siloxane, presumably originate from silicone fluids that were also used in operations (Klem 1990, Gerber et al. 1992). The origin of N-butylbenzenesulfonamide, the most frequently observed sulfurcontaining compound, is uncertain. It may be produced from benzenesulfonic acid, a constituent of the resins (Gerber et al. 1992) used during operations and butyl amine generated in ammoniarich waste.

In summary, a broad array of inorganic and organic vapors has been observed in the high-level waste single-shell tank headspaces. These vapors and their highest reported concentrations have been tabulated by functional group and concentration in this report. 


\section{References}

PL Bartley, 1999, Hazard Characterization and Analysis of Waste, Tank 241-C-106 Sluicing, Interim Report, HNF-4167, Lockheed Martin Hanford Corporation, Richland, Washington.

WL Cowley, JE Meacham, JM Grigsby, and AK Postma, 1998, Organic Solvent Topical, HNF-SD-WM-CN-032 Rev. 1, Duke Engineering and Services, Inc., Richland, Washington.

MA Gerber, LL Burger, DA Nelson, JL Ryan, and RL Zollars, 1992, Assessment of Concentration Mechanisms for Organic Wastes in Underground Storage Tanks at Hanford, PNL-8339, Pacific Northwest National Laboratory, Richland, Washington.

JL Huckaby, H Babad, and DR Bratzel, 1995, Headspace Gas and Vapor Characterization Summary for the 43 Vapor Program Suspect Tanks, WHC-SD-WM-ER-514, Rev. 1, Westinghouse Hanford Company, Richland, Washington.

JL Huckaby, JA Edwards, JC Evans, JS Fruchter, JL Julya, KB Olsen, KH Pool, BL Thomas, KL Silvers, L Jensen, LL Buckley, SR Wilmarth, and LD Pennington, and, 1995, Comparison of Vapor Sampling System (VSS) and In Situ Vapor Sampling (ISVS) Methods on Tanks C-107, BY-108, and S-102, PNNL-11186 Rev. 1, Pacific Northwest National Laboratory, Richland, Washington.

JL Huckaby, JC Evans, DS Sklarew, and AV Mitroshkov, 1998, Waste Tank Ventilation Rates Measured with a Tracer Gas Method, PNNL-11925, Pacific Northwest National Laboratory, Richland, Washington.

MJ Klem, 1990, Inventory of Chemicals Used at Hanford Site Production Plants and Support Operations (1944-1980), WHC-EP-0172, Revision 1, Westinghouse Hanford Company, Richland, Washington.

PNNL, 1999, Tank Characterization Database, Version 3.24, Pacific Northwest National Laboratory, Richland, Washington.

LA Stauffer and LM Stock, 1999, Origins of Volatile Organic Compounds Emerging from Tank 241-C-106 During Sluicing, HNF-4261, Rev. 1, Lockheed Martin Hanford Corporation, Richland, Washington.

LM Stock, 2000, The Chemistry of Flammable Gas Generation, RPP-6664, Rev. 0, CH2M Hill Hanford Group, Richland, Washington.

LM Stock and JL Huckaby, Organic Compounds in the Headspaces of Single-Shell Waste Tanks, (letter report to WL Cowley, CH2M Hill Hanford Group, Inc., March 1, 2000, from JL Huckaby, Pacific Northwest National Laboratory). 


\section{Tables of Maximum Tank Headspace Vapor Concentrations}


Table 1a. Alkanes (Page 1 of 3)

\begin{tabular}{|c|c|c|c|c|c|}
\hline Compound & $\begin{array}{c}\text { Total Number } \\
\text { of } \\
\text { Observations }\end{array}$ & $\begin{array}{c}\text { Maximum } \\
\text { Concentration, } \\
\mathrm{mg} / \mathrm{m}^{3} \\
\end{array}$ & MW & $\begin{array}{c}\text { Maximum } \\
\text { Concentration, } \\
\text { ppbv } \\
\end{array}$ & $\begin{array}{c}\text { Tank with } \\
\text { Maximum } \\
\text { Concentration }\end{array}$ \\
\hline Propane & 62 & 1.600 & $\overline{44}$ & $\overline{815}$ & BY -107 \\
\hline 2-Methyl- & 22 & 1.700 & 58 & 657 & BY-106 \\
\hline 2,2-Dimethyl- & 5 & 0.013 & 72 & 4 & U-107 \\
\hline Butane & 141 & 20.000 & 58 & 7724 & C-103 \\
\hline 2-Methyl- & 13 & 7.900 & 72 & 2458 & BY-108 \\
\hline 2,2-Dimethyl- & 9 & 0.140 & 86 & 36 & U-107 \\
\hline 2,3-Dimethyl- & 1 & 0.032 & 86 & 8 & U-107 \\
\hline 2,2,3-Trimethyl- & 1 & 0.100 & 100 & 22 & $T Y-104$ \\
\hline 2,2,3,3-Tetramethyl- & 3 & 0.240 & 114 & 47 & $B X-111$ \\
\hline Pentane & 117 & 6.500 & 72 & 2022 & BY-108 \\
\hline 2-Methyl- & 36 & 15.000 & 86 & 3907 & BY-108 \\
\hline 3-Methyl- & 24 & 2.500 & 86 & 651 & BY-108 \\
\hline 3-Ethyl- & 3 & 0.970 & 100 & 217 & $T Y-104$ \\
\hline 2,2-Dimethyl- & 1 & 0.051 & 100 & 11 & $T Y-104$ \\
\hline 2,3-Dimethyl- & 21 & 3.200 & 100 & 717 & $T Y-101$ \\
\hline 2,4-Dimethyl- & 7 & 0.550 & 100 & 123 & U-102 \\
\hline 3,3-Dimethyl- & 19 & 1.400 & 100 & 314 & TY-101 \\
\hline 3-Ethyl-2-methyl- & 1 & 0.024 & 114 & 5 & BY-106 \\
\hline 2,3,3-Trimethyl- & 4 & 1.300 & 114 & 255 & $T X-116$ \\
\hline 2,3,4-Trimethyl- & 1 & 0.290 & 114 & 57 & $T X-116$ \\
\hline Hexane & 146 & 8.600 & 86 & 2240 & $B Y-108$ \\
\hline 2-Methyl- & 21 & 3.700 & 100 & 829 & TY-101 \\
\hline 3-Methyl- & 32 & 5.600 & 100 & 1254 & TY-101 \\
\hline 2,3-Dimethyl- & 3 & 1.100 & 114 & 216 & BY -107 \\
\hline 2,4-Dimethy!- & 11 & 1.700 & 114 & 334 & $B Y-108$ \\
\hline 2,5-Dimethyl- & 5 & 0.230 & 114 & 45 & $A X-102$ \\
\hline 2,2,5-Trimethyl- & 3 & 0.060 & 128 & 11 & SX-107 \\
\hline 2,3,5-Trimethyl- & 7 & 0.300 & 128 & 53 & $B X-103$ \\
\hline $2,2,5,5$-Tetramethyl- & 1 & 0.026 & 142 & 4 & U-106 \\
\hline 3,3,4,4-Tetraethyl- & 1 & 0.082 & 142 & 13 & BY-107 \\
\hline Heptane & 165 & 4.400 & 100 & 986 & $B Y-108$ \\
\hline 2-Methyl- & 9 & 2.000 & 114 & 393 & BY -108 \\
\hline 3-Methyl- & 15 & 0.560 & 114 & 110 & BY-108 \\
\hline 3-Ethyl- & 2 & 0.038 & 128 & 7 & $T-111$ \\
\hline 4-Methyl- & 2 & 2.000 & 114 & 393 & C-103 \\
\hline 2,3-Dimethyl- & 6 & 0.280 & 128 & 49 & BY -108 \\
\hline 2,4-Dimethyl- & 10 & 0.670 & 128 & 117 & BY-101 \\
\hline 2,5-Dimethyl- & 3 & 0.110 & 128 & 19 & $\mathrm{TX}-116$ \\
\hline 2,6-Dimethyl- & 8 & 0.550 & 128 & 96 & BY-108 \\
\hline 3,5-Dimethyl- & 1 & 0.220 & 128 & 39 & BY -108 \\
\hline 2,2,4-Trimethyl- & 4 & 0.660 & 142 & 104 & $T X-116$ \\
\hline 2,3,5-Trimethyl- & 1 & 1.900 & 142 & 300 & BY-108 \\
\hline 2,3,6-Trimethyl- & 2 & 1.900 & 142 & 300 & BY-108 \\
\hline 3,3,5-Trimethyl- & 4 & 0.230 & 142 & 36 & $A X-104$ \\
\hline 2-Methyl-3-ethyl- & 7 & 0.073 & 142 & 12 & $B Y-104$ \\
\hline 2-Methyl-5-ethyl- & 1 & 0.045 & 142 & 7 & BY-102 \\
\hline 5-Methyl-3-ethyl- & 2 & 1.100 & 142 & 174 & C-102 \\
\hline 4-(1-methylethyl)- & 2 & 0.440 & 170 & 58 & BY-108 \\
\hline 4-Ethyl-2,2,6,6-tetramethyl- & $\overline{2}$ & 0.370 & 184 & 45 & $A X-104$ \\
\hline $2,2,3,4,6,6$-Hexamethyl- & 4 & 0.240 & 184 & 29 & B-103 \\
\hline Octane & 137 & 1.800 & 114 & 354 & $C-103$ \\
\hline 3-Methyl- & 6 & 0.620 & 128 & 109 & BY -108 \\
\hline 4-Methyl- & 6 & 1.200 & 128 & 210 & $c-102$ \\
\hline 4-Ethyl- & 2 & 0.070 & 142 & 11 & BY -109 \\
\hline 2,5-Dimethyl- & 2 & 0.330 & 142 & 52 & C-101 \\
\hline 2,6-Dimethyl- & 6 & 0.330 & 142 & 52 & BY -107 \\
\hline 3,5-Dimethyl- & 2 & 0.160 & 142 & 25 & BY-104 \\
\hline 2,3,3-Trimethyl- & 1 & 0.700 & 156 & 101 & BY -107 \\
\hline 2,3,7-Trimethyl- & 4 & 3.100 & 156 & 445 & BX-104 \\
\hline 2,5,6-Trimethyl- & 2 & 0.031 & 156 & 4 & U-107 \\
\hline 2-Methyl-5-ethyl- & 1 & 0.180 & 156 & 26 & C-101 \\
\hline 2-Methyl-6-ethyl- & 5 & 0.290 & 156 & 42 & $B Y-108$ \\
\hline
\end{tabular}


Table 1a. Alkanes (Page 2 of 3)

Compound

Nonane
2-Methyl-
3-Methyl-
4-Methyl-
2,6-Dimethyl-
4,5-Dimethyl-
3,7-Dimethyl-
3-Methyl-5-propyl-
5-(2-Methylpropyl)-
5-Butyl-

2-Methyl-

3-Methyl-

4-Methyl-

5-Methyl-

5-Propyl-

2,5-Dimethyl-

2,9-Dimethyl-

3,8-Dimethyl-

2,2,8-Trimethyl-

2,3,5-Trimethyl-

2,3,6-Trimethy!-

2,3,7-Trimethyl-

2,3,8-Trimethyl-

2,4,6-Trimethy!-

2,5,9-Trimethyl-

2,6,7-Trimethyl-

2,6,8-Trimethyl-

2-Methyl-6-ethyl-

2,3,5,8-Tetramethyl-

Undecane

2-Methyl-

3-Methyl-

4-Methyl-

5-Methyl-

5-Ethyl-

6-Methyl-

2,3-Dimethyl-

2,4-Dimethyl-

2,5-Dimethyl-

2,6-Dimethyl-

2,7-Dimethyl-

2,8-Dimethyl-

2,9-Dimethyl-

2,10-Dimethyl-

3,4-Dimethyl-

3,6-Dimethyl-

3,7-Dimethyl-

3,8-Dimethyl-

3,9-Dimethyl-

4,4-Dimethyl-

4,6-Dimethyl-

4,7-Dimethyl-

4,8-Dimethyl-

5,5-Dimethyl-

5,7-Dimethyl-

Dodecane

6,6-Dimethyl-

2-Methyl-

3-Methyl-

4-Methyl-

5-Methyl-
Total Number

of

Observations

141
2

3

11

2
2

2
6

6

2

4
147

16

9

16

5

3

3

2

5

2

3

6
3

3
4

4
3

1

4

2

1
156

16

14

19

14

5
10

3

10

1

29

2

4

2

14

3

9

5

2

4

3

5

1
3

1

156

3
5

15

3
Maximum Concentration, $\mathrm{mg} / \mathrm{m}^{3}$

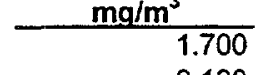

0.130

0.240

0.710

4.200

0.009

2.400

0.660

0.057

0.340

18.000

2.100

1.100

1.000

0.890

0.028

3.200

0.014

0.300

0.280

0.230

0.710

1.000

0.260

24.000

0.029

1.400

0.014

2.200

0.160

92.000

28.000

18.000

13.000

6.900

1.300

3.500

0.970

15.000

0.099

41.000

0.600

0.850

0.240

39.000

0.710

0.100

22.000

4.200

0.190

0.570

5.200

2.300

0.500

1.400

0.810

0.890

388.00

3.000

1.800

19.000

0.570

\begin{tabular}{c}
$M W$ \\
\hline 128 \\
142 \\
142 \\
142 \\
156 \\
156 \\
156 \\
184 \\
184 \\
184 \\
142
\end{tabular}

Maximum

Concentration, ppbv

298
21
38
112

112

603
1

345

80

7

41
2839

302

158

144

128

3

422

2

40

34

28

86

122

32

2922

4

170

2

268

18
13210

3689

2372

1713

909

158

461

118

1826

12

4991

73

103

29

4748

86

12

2678

511

23

69

633

280

61

170

99

108

51125

365

219

2313

69

Tank with

Maximum

Concentration

C-103

BY-107

BY -108

C-102

C-103

BY-105

BY -108

U-106

BY -110

BY-107

C-103

C-102

C-102

C-204

C-102

T-107

C-101

A-102

BX-104

U-106

BY -109

BY -108

BX-104

BY -107

C-103

BY-109

BY-104

TY-104

C-102

BY-108

C-103

C-103

C-103

C-103

C-103

C-102

C-102

BY-108

C-103

C-110

C-103

BY -108

BY-107

BY-107

C-103

TY-103

BY -107

C-103

C-102

A-106

TY-103

C-101

BY-108 
Table 1a. Alkanes (Page 3 of 3)

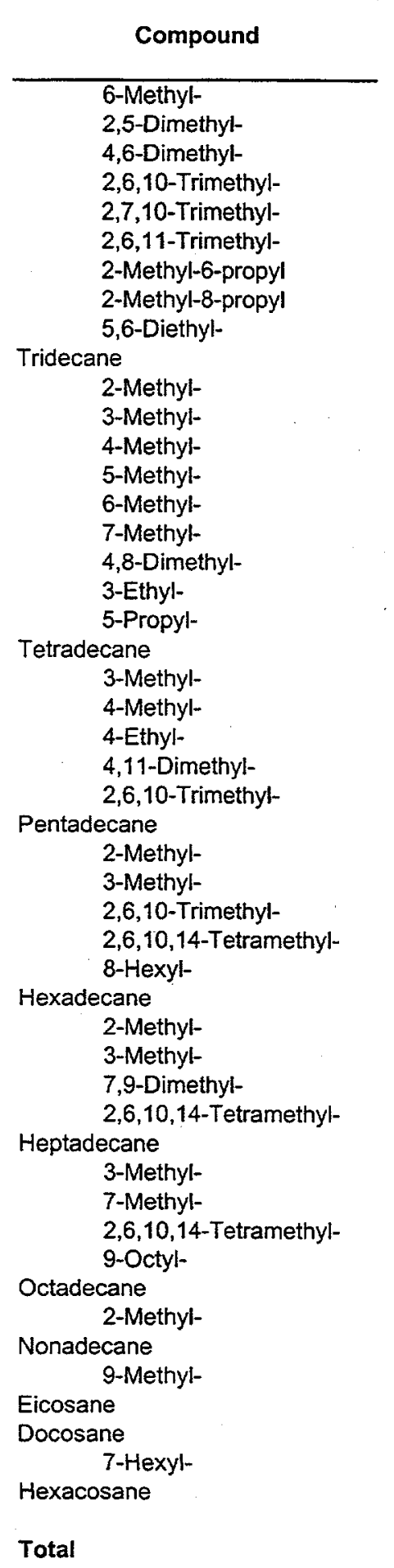

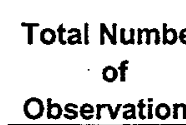

Observations

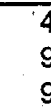

9
16

6

11

4
8

8
2

174

22

10

13

4

14

16

9

3
4

134

5

4

1

3

4

48

6

1

5

5

2
29

2

2

3

9

20

1

1

1

1

8

2

3

2

6

1
1

2731

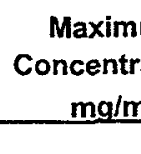

0.790
2.500

62.000

59.000

5.300

48.000

0.040

2.900

0.076

508.000

21.000

12.000

1.500

12.000

29.000

12.000

2.200

1.100

1.900

121.000

0.380

3.100

0.040

0.010

0.190

37.000

0.330

0.064

0.094

0.057

0.072

34.000

0.067

0.440

0.012

0.310

44.000

0.051

0.500

0.046

0.270

0.074

0.910

0.003

0.012

0.210

0.061

0.030

1915.2

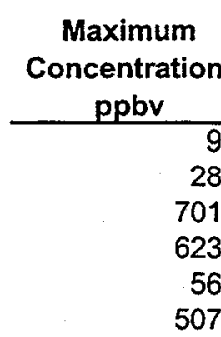

4
287

8

61843

2376

1358

170

1358

3281

1358

232

116

188

13689

40

328

4

1
18

3909

33

226

226

254

268

296

226

240

240

254

282

240

254

254

296

352

254

268

268

282

296

394

366

2

249,865
Tank with

Maximum

Concentration

BY-108

BY-108

C-103

C-103

C-102

C-103

BY-104

BY-108

BY-107

C-103

C-103

C-103

BY-108

C-103

C-103

BY-108

BY-108

BY-107.

BY-107

C-103

BY-108

BX-104

BY-104

BY-105

BY-107

C-103

C-102

C-110

BY-107

BY-107

BY-107

C-103

BY-107

BY-107

B-103

BY -110

C-103

C-110

BY-108

BY -107

TY-103

BY-108

TY-103

C-107

B-103

BY -107

C. -110

BY -108 
Table 1b. Alkanes Observed in One Tank at Concentration Less Than $0.025 \mathrm{mg} / \mathrm{m}^{3}$

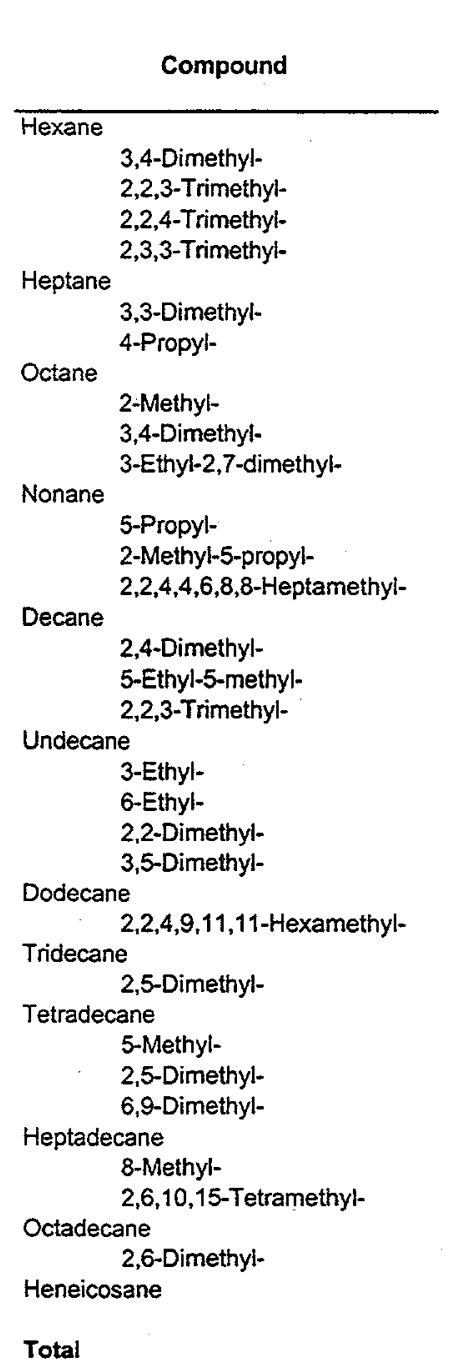

\begin{tabular}{|c|c|c|}
\hline $\begin{array}{c}\text { Total Number } \\
\text { of } \\
\text { Observations } \\
\end{array}$ & $\begin{array}{c}\text { Maximum } \\
\text { Concentration, } \\
\mathrm{mg}^{2} \mathrm{~m}^{3} \\
\end{array}$ & MW \\
\hline 1 & 0.005 & 114 \\
\hline 1 & 0.019 & 128 \\
\hline 1 & 0.012 & 128 \\
\hline 1 & 0.002 & 128 \\
\hline 1 & 0.019 & 128 \\
\hline 1 & 0.010 & 142 \\
\hline 1 & 0.020 & 128 \\
\hline 1 & 0.009 & 142 \\
\hline 1 & 0.014 & 170 \\
\hline 1 & 0.004 & 170 \\
\hline 1 & 0.002 & 184 \\
\hline 1 & 0.013 & 226 \\
\hline 1 & 0.003 & 170 \\
\hline 1 & 0.010 & 184 \\
\hline 1 & 0.014 & 184 \\
\hline 1 & 0.002 & 184 \\
\hline 1 & 0.003 & 184 \\
\hline 1 & 0.016 & 184 \\
\hline 1 & 0.001 & 184 \\
\hline 1 & 0.016 & 254 \\
\hline 1 & 0.009 & 212 \\
\hline 1 & 0.002 & 212 \\
\hline 1 & 0.010 & 226 \\
\hline 1 & 0.001 & 226 \\
\hline 1 & 0.002 & 254 \\
\hline 1 & 0.008 & 296 \\
\hline 1 & 0.002 & 282 \\
\hline 1 & 0.013 & 296 \\
\hline 28 & 0.240 & \\
\hline
\end{tabular}

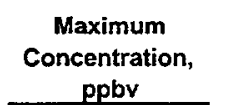

ppov

1
3
2
0.4

3

1

4

4
1
2

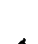

0.2

1

0.4
1
2

2

0.3

0.4

2

0.1

1

1

0.2

1

0.1

0.2

1

0.2

1

32

Tank with
Maximum
Concentration

C-107

U-107

TY-104

T-107

C- 110

A-102

BY-106

BY-105

U-107

C-104

T-107

S-105

BY-106

TX-118

AX-102

TY-104

BY-106

U-107

C-112

S-105

BY-103

T-107

BY-103

T-107

C-107

C-107

T-107

C-107
$75163-97-2$
$629-94-7$

TWINS2

(CAS)

Number

583-48-2M

16747-25-4

$\{6747-26-5$

16747-28-7

4032-86-4

3178-29-8

3221-61-2

15869-92-8

62183-55-5

998-35-6

31081-17-1

4390-04-9

2801-84-5

17312-74-2

62338-09-4

17312-58-2

17312-60-6

17312-64-0

17312-81-1

6304-50-3

56292-66-1

25117-32-2

56292-69-4

55045-13-1

13287-23-5

54833-48-6

$629-94-7$ 
Table 2a. Cycloalkanes (Page 1 of 2)

\begin{tabular}{|c|c|c|c|c|c|c|}
\hline Compound & & $\begin{array}{c}\text { Total Number } \\
\text { of } \\
\text { Observations }\end{array}$ & $\begin{array}{c}\text { Maximum } \\
\text { Concentration, } \\
\mathrm{mg} / \mathrm{m}^{3}\end{array}$ & MW & $\begin{array}{c}\text { Maximum } \\
\text { Concentration, } \\
\text { ppbv }\end{array}$ & $\begin{array}{l}\text { Tank with } \\
\text { Maximum } \\
\text { Concentration }\end{array}$ \\
\hline \multicolumn{7}{|l|}{ CYCLOALKANES } \\
\hline Cyclopropane & & 38 & 0.380 & 42 & 203 & BY-107 \\
\hline Methyl- & & 1 & 0.170 & 56 & 68 & C-104 \\
\hline Ethyl- & & 5 & 7.300 & 70 & 2336 & $B Y-108$ \\
\hline 1,1-Dimethyl- & & 4 & 0.510 & 70 & 163 & BY-107 \\
\hline trans-1,2-Dimethyl- & & 1 & 0.049 & 70 & 16 & BY-110 \\
\hline Propyl- & & 6 & 0.650 & 84 & 173 & C-102 \\
\hline (1-Methylethyl)- & & 3 & 0.290 & 84 & 77 & BY-104 \\
\hline Butyl & & 5 & 2.500 & 98 & 571 & BY-108 \\
\hline (1-Methylbutyl)- & & 1 & 0.029 & 112 & 6 & $B Y-110$ \\
\hline Pentyl- & & 3 & 0.060 & 112 & 12 & BY-109 \\
\hline Octyi- & & 2 & 1.200 & 154 & 175 & C-102 \\
\hline 1,2-Dibutyl- & $\left({ }^{*}\right)$ & 1 & 0.042 & 154 & 6 & BY-110 \\
\hline 1-Pentyl-2-propyl- & $\left(*^{*}\right)$ & 1 & 1.600 & 154 & 233 & C-101 \\
\hline 1-Ethyl-2-heptyl- & $(*)$ & 1 & 0.060 & 168 & 8 & BY-104 \\
\hline Cyclobutane & & 3 & 0.045 & 56 & 18 & BY-107 \\
\hline Methyl- & & 1 & 1.400 & 70 & 448 & $U-103$ \\
\hline Ethyl- & & 1 & 0.570 & 84 & 152 & BY -107 \\
\hline (1-Methylethyl)- & & 3 & 0.160 & 98 & 37 & BY -109 \\
\hline cis-1,2-Diethyl- & $\left({ }^{* *}\right)$ & 2 & 0.320 & 112 & 64 & C-102 \\
\hline trans-1,2-Diethy\}- & & 2 & 0.460 & 112 & 92 & C-101 \\
\hline Cyclopentane & & 5 & 0.650 & 70 & 208 & BY-107 \\
\hline Methyl- & & 12 & 2.100 & 84 & 560 & BY -108 \\
\hline Ethyl- & & 3 & 0.040 & 98 & 9 & BY-104 \\
\hline cis-1,2-Dimethyl- & $(* *)$ & 5 & 0.340 & 98 & 78 & S-103 \\
\hline trans-1,2-Dimethyl- & & 8 & 0.490 . & 98 & 112 & BY-108 \\
\hline cis-1,2-Dimethyi-trans-3-methyl- & & 3 & 0.032 & 112 & 6 & BY-107 \\
\hline 1,1,3-Trimethyl- & $\left({ }^{*}\right)$ & 2 & 0.360 & 112 & 72 & BY-108 \\
\hline 1,2,4-Trimethyl- & $\left({ }^{\star}\right)$ & 1 & 0.180 & 112 & 36 & S-103 \\
\hline$a, a, b-1,2,4-$ Trimethyi- & & 4 & 0.030 & 112 & 6 & BY-104 \\
\hline (1-Methylbutyl)- & & 2 & 0.240 & 140 & 38 & BY -107 \\
\hline (2-Methylbutyl)- & & 2 & 0.350 & 140 & 56 & BY-108. \\
\hline 1,2-Dimethyl-3-(1-methylethyl)- & $\left({ }^{\star}\right)$ & 2 & 0.320 & 140 & 51 & BY-107 \\
\hline 1,3-Dimethyl-2-(1-methylethyl)- & $\left(^{*}\right)$ & 5 & 0.760 & 140 & 122 & BY-108 \\
\hline 1-Methyl-3-(2-methylpropyl)- & $(\star)$ & 5 & 0.570 & 140 & 91 & BY-108 \\
\hline 1-Butyl-2-ethyl- & $\left({ }^{\star}\right)$ & 2 & 0.110 & 154 & 16 & BY-107 \\
\hline 1-Butyl-2-propyl- & $\left({ }^{\star}\right)$ & 1 & 0.160 & 168 & 21 & BY -107 \\
\hline 1-Hexyl-3-methyl- & $(*)$ & 6 & 0.270 & 168 & 36 & $C-110$ \\
\hline 1-Pentyl-2-propyl- & $\left({ }^{*}\right)$ & 4 & 0.470 & 182 & 58 & C-102 \\
\hline Decyl & & 1 & 0.530 & 210 & 57 & C-103 \\
\hline Cyclohexane & & 54 & 4.300 & 84 & 1147 & C-103 \\
\hline Methyl- & & 41 & 1.600 & 98 & 366 & TY-110 \\
\hline Ethyl- & & 5 & 0.460 & 112 & 92 & $B Y-108$ \\
\hline 1,2-Dimethyl- & $(*)$ & 1 & 0.250 & 112 & 50 & BY-108 \\
\hline cis-1,3-Dimethyl- & & 4 & 0.340 & 112 & 68 & C-204 \\
\hline 1,4-Dimethyl- & $(*)$ & 2 & 0.280 & 112 & 56 & C-204 \\
\hline Cyclopropyl- & & 3 & 0.280 & 124 & 51 & BY-108 \\
\hline Propyl- & & 3 & 0.110 & 126 & 20 & C-102 \\
\hline 1,1,2-Trimethyl- & $\left({ }^{*}\right)$ & 2 & 1.500 & 126 & 267 & $\mathrm{C}-204$ \\
\hline 1,1,3-Trimethyl- & $(*)$ & 10 & 0.810 & 126 & 144 & BY-108 \\
\hline 1,2,3-Trimethyl- & $(*)$ & 2 & 0.003 & 126 & 1 & $T-107$ \\
\hline 1,3,5-Trimethyl- & $\left({ }^{*}\right)$ & 2 & 0.009 & 126 & 2 & BY-106 \\
\hline Butyl- & & 6 & 1.500 & 140 & 240 & BY -108 \\
\hline (1-Methylpropyl)- & & 5 & 2.100 & 140 & 336 & C-103 \\
\hline cis-1-Ethyl-4-methyl- & & 6 & 0.570 & 140 & 91. & BX-104 \\
\hline trans-1-Ethyl-4-methyl- & & 5 & 0.170 & 140 & 27 & BY-108 \\
\hline 1-Methyi-2-propyl- & $\left({ }^{*}\right)$ & 2 & 0.078 & 140 & 12 & $T-111$ \\
\hline 1-Methyl-3-propyl- & $(*)$ & 4 & 0.590 & 140 & 94 & C-102 \\
\hline 1-Methyl-3-(1-methylethyl)- & $(*)$ & 1 & 0.340 & 140 & 54 & C-110 \\
\hline cis-1-Methyl-4-(1-methylethyl)- & & 3 & 0.350 & 140 & 56 & BY -108 \\
\hline $1,1,2,3-$ Tetramethyl- & $(*)$ & 10 & 0.400 & 140 & 64 & C-204 \\
\hline cis-1,1,3,5-Tetramethyl- & & 3 & 0.460 & 140 & 74 & $B X-104$ \\
\hline trans-1,1,3,5-Tetramethyl- & & 2 & 0.021 & 140 & 3 & C- 110 \\
\hline 1,1,4,4-Tetramethyl- & $(*)$ & 1 & 0.410 & 140 & 66 & BY -108 \\
\hline$a, a, a, a-1,2,4,5$-Tetramethyl- & & 1 & 0.051 & 140 & 8 & $C-110$ \\
\hline (1,2-Dimethylpropyl)- & & 1 & 0.170 & 154 & 25 & BY -107 \\
\hline Pentyl- & & 21 & 2.400 & 154 & 349 & C-204 \\
\hline 1-Ethyl-2-propyl- & $\left({ }^{*}\right)$ & 3 & 0.430 & 154 & 63 & BY-108 \\
\hline 1,1-Dimethyl-2-propyl- & $\left({ }^{*}\right)$ & 5 & 0.300 & 154 & 44 & $C-102$ \\
\hline
\end{tabular}


Table 2a. Cycloalkanes (Page 2 of 2)

\begin{tabular}{|c|c|c|c|c|c|c|}
\hline Compound & & $\begin{array}{c}\text { Total Number } \\
\text { of } \\
\text { Observations }\end{array}$ & $\begin{array}{c}\text { Maximum } \\
\text { Concentration, } \\
\mathrm{mg} / \mathrm{m}^{3}\end{array}$ & MW & $\begin{array}{c}\text { Maximum } \\
\text { Concentration, } \\
\text { ppbv }\end{array}$ & $\begin{array}{c}\text { Tank with } \\
\text { Maximum } \\
\text { Concentration }\end{array}$ \\
\hline 1-Ethyl-2,2,6-trimethyl- & $(*)$ & $\overline{2}$ & $\overline{0.550}$ & 154 & $\overline{80}$ & $\overline{B Y-108}$ \\
\hline (3-Methylpentyl)- & & 1 & 0.650 & 168 & 87 & C-102 \\
\hline (4-Methylpentyl) - & & 5 & 2.700 & 168 & 360 & $B Y-108$ \\
\hline Hexyl- & & 14 & 0.670 & 168 & 89 & BY-107 \\
\hline 1-Methyl-2-pentyl- & $(*)$ & 3 & 0.530 & 168 & 71 & BY-108 \\
\hline 1-Methyl-2-pentyl- & $\left({ }^{*}\right)$ & 3 & 0.530 & 168 & 71 & BY -108 \\
\hline 1-Methyl-3-pentyl- & $(*)$ & 3 & 0.077 & 168 & 10 & C- 110 \\
\hline 1-Methyl-4-(1-methylbutyl)- & $(*)$ & 4 & 0.130 & 168 & 17 & BY -107 \\
\hline 1,2-Diethyl-1-methyl- & $\left(^{*}\right)$ & 4 & 0.360 & 168 & 48 & BY -108 \\
\hline 1,2-Diethyl-3-methyl- & $(*)$ & 4 & 0.470 & 168 & 63 & BY-108 \\
\hline 2,4-Diethyl-1-methyl- & $(*)$ & 4 & 0.280 & 168 & 37 & BY -107 \\
\hline 1,5-Diethyl-2,3-dimehtyl- & $(*)$ & 2 & 0.570 & 168 & 76 & C-102 \\
\hline (2,2-Dimethylcyclopentyl)- & & 4 & 0.310 & 180 & 39 & $B X-104$ \\
\hline 2-Butyl-1,1,3-trimethyl- & $\left({ }^{*}\right)$ & 18 & 3.400 & 182 & 418 & C-102 \\
\hline Octyl- & & 6 & 0.130 & 206 & 14 & B-103 \\
\hline (1-Methylheptyl)- & $\left({ }^{* * *}\right)$ & 1 & 2.600 & 206 & 283 & BY-108 \\
\hline 1,1,3-Trimethyl-2-(3-methylpentyl & $(*)$ & 5 & 0.740 & 210 & 79 & $B X-104$ \\
\hline cis-(1-Cyclohexylmethyl)-2-methyl & & 1 & 0.110 & 232 & 11 & BY -107 \\
\hline trans-(1-Cyclohexylmethyl)-2-mett & thyl- & 1 & 0.250 & 232 & 24 & BY-108 \\
\hline cis-(1-Cyclohexylmethyl)-4-methyl & & 1 & 0.800 & 232 & 77 & BY-108 \\
\hline (2-Ethyloctyl) - & $(* * *)$ & 3 & 0.048 & 234 & 5 & BY-104 \\
\hline cis-(1-Cyclohexylmethyl)-2-ethyl- & & 3 & 1.000 & 246 & 91 & BY -108 \\
\hline 1,3,5,-Trimethyl-2-octadecyl- & $(*)$ & 2 & 0.140 & 378 & 8 & BY -104 \\
\hline Cyclooctane & & & & & & \\
\hline Butyl- & & 1 & 0.330 & 168 & 44 & $C-102$ \\
\hline cis-1,4-Dimethyl- & & 1 & 0.040 & 140 & 6 & BY-104 \\
\hline Cycloundecane & & & & & & \\
\hline 1,1,2-Trimethyl- & $\left({ }^{*}\right)$ & 1 & 0.042 & 196 & 5 & BY -109 \\
\hline Cyclododecane & & 7 & 2.700 & 168 & 360 & C-102 \\
\hline Ethyl- & & 1 & 0.027 & 206 & 3 & TY-103 \\
\hline Cyclhexadecane & & 4 & 0.100 & 224 & 10 & $S-110$ \\
\hline DECALINS & & & & & & \\
\hline trans-Decahydronaphthalene & & 18 & 4.700 & 138 & 763 & C-103 \\
\hline 2-Methyl- & $\left({ }^{*}\right)$ & 17 & 21.000 & 152 & 3095 & C-103 \\
\hline 1,2-Dimethyl- & $(*)$ & 1 & 1.400 & 168 & 187 & C-102 \\
\hline 1,5-Dimethyl- & $(*)$ & 3 & 0.029 & 168 & 4 & $B Y-110$ \\
\hline 1,6-Dimethyl- & $\left({ }^{*}\right)$ & 5 & 0.360 & 168 & 48 & BY-108 \\
\hline 2,3-Dimethyl- & $\left({ }^{*}\right)$ & 7 & 3.100 & 168 & 413 & C-204 \\
\hline 2,6-Dimethyl- & $(*)$ & 8 & 2.500 & 168 & 333 & C-204 \\
\hline 2-Butyl- & $(*)$ & 1 & 0.320 & 180 & 40 & $B \gamma-108$ \\
\hline INDANES & & & & & & \\
\hline $\begin{array}{l}\text { cis-Octahydro- } 1 \mathrm{H} \text {-indene } \\
\text { trans-Octahydro- } 1 \mathrm{H} \text {-indene }\end{array}$ & & 1 & 0.037 & 124 & 7 & BY-110 \\
\hline $2,2,4,4,7,7$-Hexamethyl- & & 1 & 0.200 & 208 & 22 & BY-107 \\
\hline OTHER BICYCLIC AND TRICYCLIC MOL & LEC & ULES & & & & \\
\hline 1,1'-Bicyclohexyl & & 1 & 0.046 & 168 & 6 & $B Y-107$ \\
\hline cis-2-Methyl- & & 1 & 0.120 & 182 & 15 & $B X-104$ \\
\hline Bicyclo[2.2.1]heptane & & 3. & 0.020 & 96 & 5 & BY-107 \\
\hline Bicyclo[4.1.0]heptane & & & & & & \\
\hline 2-Methyl-7-pentyl- & $\left({ }^{*}\right)$ & 1 & 1.300 & 180 & 162 & C-204 \\
\hline 3-Methyl-7-pentyl- & $\left({ }^{*}\right)$ & 1. & 0.410 & 180 & 51 & $B Y-108$ \\
\hline Bicyclo[2.2.2]octane & & & & & & \\
\hline $1,2,3,6$-Tetramethyl- & $\left({ }^{\star}\right)$ & 1 & 0.060 & 166 & 8 & C-110 \\
\hline Bicyclo[3.3.1]nonane & & 1 & 0.047 & 124 & 8 & $\mathrm{~T}-111$ \\
\hline Total & & 545 & 102.0 & & 18,010 & \\
\hline
\end{tabular}

Notes

(*) The positional, geometrical or stereo isomer has not been defined.

$\left.{ }^{\star \star}\right)$ The concentration of the compound has been estimated from results for a mixture of isomeric compounds.

$\left.{ }^{(* * *}\right)$ The name used in this table differs from the name used in the data base. 
Table 2b. Cycloalkanes Observed in One Tank at Concentration Less Than $0.025 \mathrm{mg} / \mathrm{m}^{3}$

\begin{tabular}{|c|c|c|c|c|c|c|c|}
\hline Compound & \multicolumn{2}{|r|}{$\begin{array}{c}\text { Total Number } \\
\text { of } \\
\text { Observations }\end{array}$} & \multirow{2}{*}{$\begin{array}{c}\begin{array}{c}\text { Maximum } \\
\text { Concentration, } \\
\mathrm{mg} / \mathrm{m}^{3}\end{array} \\
\end{array}$} & \multirow[t]{2}{*}{ MW } & \multirow[t]{2}{*}{$\begin{array}{c}\text { Maximum } \\
\text { Concentration, } \\
\text { ppbv }\end{array}$} & \multirow[t]{2}{*}{$\begin{array}{c}\text { Tank with } \\
\text { Maximum } \\
\text { Concentration } \\
\end{array}$} & \multirow[t]{2}{*}{$\begin{array}{l}\text { TWINS2 } \\
\text { (CAS) } \\
\text { Number } \\
\end{array}$} \\
\hline $\begin{array}{l}\text { CYCLOALKANES } \\
\text { Cyclopropane }\end{array}$ & & & & & & & \\
\hline cis-1,2-Dimethyl- & & 1 & 0.0170 & 70 & 5 & C-107 & $930-18-7$ \\
\hline trans-1-Butyl-2-methyl- & & 1 & 0.0110 & 112 & 2 & B-103 & $38851-70-6$ \\
\hline 1-Ethyl-2-pentyl- & $(*)$ & 1 & 0.0013 & 140 & 0.2 & T-107 & $62238-08-8$ \\
\hline 1,1,2-Trimethyl-3-(2-methylpropyl)- & & 1 & 0.0100 & 140 & 2 & BY-105 & $41977-43-9$ \\
\hline 1-Heptyl-2-methyl- & $(*)$ & 1 & 0.0150 & 154 & 2 & BY-106 & $74663-91-5$ \\
\hline 1-Butyl-1-methyl-2-propyl- & & 1 & 0.0055 & 154 & 1 & BY-105 & $41977-34-8$ \\
\hline 1-(2-Butyl)-1-(2-methylbutyl)- & $\left({ }^{*}\right)$ & 1 & 0.0065 & 168 & 1 & $T Y-104$ & UCY012-08 \\
\hline Cyclobutane & & & & & & & \\
\hline $\begin{array}{l}\text { 1,1,2,3,3-Pentamethyl- } \\
\text { Cyclopentane }\end{array}$ & & 1 & 0.0080 & 126 & 1 & C-108 & $57905-86-9$ \\
\hline cis-1,3-Dimethyl- & & 1 & 0.0060 & 98 & 1 & BY-106 & $2532-58-3$ \\
\hline cis-1-Ethyl-3-methyl- & & 1 & 0.0006 & 112 & 0.1 & $T Y-104$ & $2613-66-3$ \\
\hline 1,2,3- Trimethyl- & $(*)$ & 1 & 0.0048 & 112 & 1 & $A X-102$ & $2815-57-8$ \\
\hline (2-Methyipropyl)- & & 1 & 0.0250 & 126 & 4 & TY-118 & $3788-32-7$ \\
\hline 2-Ethyl-1,1-dimethyl- & & 1 & 0.0022 & 126 & 0.4 & $5 X-106$ & $54549-80-3$ \\
\hline cis-1,1,3,4- Tetramethy!- & & 1 & 0.0200 & 126 & 4 & BY-104 & $53907-60-1$ \\
\hline trans-1-Methyl-2-(4-methylpentyi)- & & 1 & 0.0006 & 168 & 0.1 & $T Y-104$ & $66553-50-2$ \\
\hline 3-Hexyl-1,1-dimethyl- & & 1 & 0.0035 & 182 & 0.4 & TY-104 & $61142-65-2$ \\
\hline Cyclohexane & & $\cdots$ & & & & & \\
\hline 1,7-Dimethyl- & & 1 & 0.0047 & 112 & 1 & $T X-118$ & $590-66-9$ \\
\hline (1-Methylethyl)- & & 1 & 0.0070 & 126 & 1 & $B Y-106$ & $696-29-7$ \\
\hline cis-1-Ethyl-2-methyl- & & 1 & 0.0160 & 126 & 3 & $B Y-106$ & $4923-77-7$ \\
\hline a,b,b-1,2,4-Trimethyl- & & 1 & 0.0140 & 126 & 2 & $T-111$ & $7667.60-9$ \\
\hline (2-Methylpropyl)- & & 1 & 0.0092 & 140 & 1 & $A-102$ & $1678-98-4$ \\
\hline 1-Methyl-4-(1-methylethyl)- & $(*)$ & 1 & 0.0230 & 140 & 4 & $T-111$ & $99-82-1$ \\
\hline 1,2,4,5-Tetramethyl- & $(*)$ & 1 & 0.0100 & 140 & 2 & BY-105 & $2090-38-2$ \\
\hline 1,2-Diethyl-1-methyl- & & 1 & 0.0050 & 154 & 1 & BY-106 & $61141-79-5$ \\
\hline (1,2-Dimethylbutyl)- & & 1 & 0.0083 & 168 & 1 & B-103 & $61142-37-8$ \\
\hline 2-Propyl-1,1,3-trimetnyl- & $\left({ }^{*}\right)$ & 1 & 0.0060 & 168 & 1 & $B Y-106$ & UCY012-04 \\
\hline 1,2-Dimethyl-3-pentyl- & & 1 & 0.0093 & 182 & 1 & B-112 & UCY013-03 \\
\hline trans-1-(Cyclohexyimethyl)-4-methy- & & 1 & 0.0100 & 196 & 1 & BY-105 & $54823-98-2$ \\
\hline cis-1-(Cyclohexylmethyl)-4-ethyl- & & 1 & 0.0047 & 210 & 1 & C-109 & $54934-95-1$ \\
\hline (1-Propylheptyl)- & & 1 & 0.0020 & 224 & 0.2 & C-111 & $13151-75-2$ \\
\hline Decyl- & & 1 & 0.0020 & 224 & 0.2 & BY-105 & $1795-16-0$ \\
\hline Cyclooctane & & 1 & 0.0060 & 112 & 1. & TY-104 & $292-64-8$ \\
\hline 1,5-Dimethyl- & $(*)$ & 1 & 0.0100 & 140 & 2 & BY-105 & $21328-57-4$ \\
\hline Cyclotetradecane & & 1 & 0.0270 & 196 & 3 & $T-111$ & $295-17-0 M$ \\
\hline OTHER BICYCLIC AND TRICYCLIC MOLECU & JLES & & & & & & \\
\hline $\begin{array}{l}\text { 1,1-Bicyclohexyl } \\
\text { trans-2-methyl- }\end{array}$ & & 1 & 0.0220 & 180 & 3 & C-110 & 50991-09-8 \\
\hline Bicyclo[4.1.0]heptane & & 1 & 0.0230 & 96 & 5 & $\mathrm{C}-110$ & $286-08-8$ \\
\hline Total & & 36 & 0.3562 & & 60 & & \\
\hline
\end{tabular}

Note

(*) The positional, geometrical or stereo isomer has not been defined. 
Table 3a. Alkenes and Alkadienes (Page 1 of 3)

\begin{tabular}{|c|c|c|c|c|c|c|}
\hline Compound & & $\begin{array}{c}\begin{array}{c}\text { Total Number } \\
\text { of }\end{array} \\
\text { Observations } \\
\end{array}$ & $\begin{array}{c}\begin{array}{c}\text { Maximum } \\
\text { Concentration, } \\
\mathrm{mg} / \mathrm{m}^{3}\end{array} \\
\end{array}$ & MW & $\begin{array}{c}\text { Maximum } \\
\text { Concentration, } \\
\text { ppbv }\end{array}$ & $\begin{array}{c}\text { Tank with } \\
\text { Maximum } \\
\text { Concentration }\end{array}$ \\
\hline \multicolumn{7}{|l|}{ ALKENES } \\
\hline Propene & & 42 & 8.300 & 42 & 4427 & $B Y-108$ \\
\hline 2-Methyl- & & 70 & 16.000 & 56 & 6400 & C-103 \\
\hline 1-Butene & & 28 & 23.000 & 56 & 9200 & C-103 \\
\hline 2-Methyl- & & 4 & 0.210 & 70 & 67 & BY-108 \\
\hline 3-Methyl- & & 4 & 1.500 & 70 & 480 & BY-108 \\
\hline 2,3-Dimethyl- & & 1 & 0.025 & 84 & 7 & BY-105 \\
\hline 3,3-Dimethyl- & & 1 & 0.032 & 84 & 9 & $U-106$ \\
\hline \multicolumn{7}{|l|}{ 2-Butene } \\
\hline 2-Methyl- & & 3 & 0.160 & 70 & 51 & BY-104 \\
\hline 2,3-Dimethyl- & & 1 & 0.130 & 84 & 35 & BY-104 \\
\hline E-2-Butene & $\left({ }^{*}\right)$ & 5 & 0.130 & 56 & 52 & C-104 \\
\hline Z-2-Butene & $\left({ }^{*}\right)$ & 5 & 1.600 & 56 & 640 & $C-103$ \\
\hline 1-Pentene & & 18 & 10.000 & 70 & 3200 & $C-103$ \\
\hline 2-Methyl- & & 6 & 2.600 & 84 & 693 & BY-108 \\
\hline 4-Methyl- & & 14 & 2.500 & 84 & 667 & BY-108 \\
\hline 3,4-Dimethyl- & & 2 & 1.800 & 98 & 411 & BY -108 \\
\hline 4,4-Dimethyl- & & 2 & 0.050 & 98 & 11 & $T X-111$ \\
\hline \multicolumn{7}{|l|}{ 2-Pentene } \\
\hline 2,4-Dimethyl- & & 2 & 0.037 & 98 & 8 & B-103 \\
\hline E-2-Pentene & $\left({ }^{*}\right)$ & 4 & 0.950 & 70 & 304 & BY -107 \\
\hline 4-Methyl- & & 1 & 0.028 & 84 & 7 & BY -106 \\
\hline Z-2-Pentene & $(*)$ & 11 & 1.300 & 70 & 416 & BY-108 \\
\hline 1-Hexene & & 49 & 13.000 & 84 & 3467 & C-103 \\
\hline 4-Methyl- & & 1 & 0.440 & 98 & 101 & $S-103$ \\
\hline 5-Methyl- & & 3 & 1.600 & 98 & 366 & BY-108 \\
\hline 4-Ethyl- & & 1 & 0.210 & 112 & 42 & S-103 \\
\hline 3,4-Dimethyl- & & 4 & 0.340 & 112 & 68 & BY-107 \\
\hline 4,5-Dimethyl- & & 3 & 0.340 & 112 & 68 & BY-108 \\
\hline 5,5-Dimethyl- & & 2 & 0.018 & 112 & 4 & C-110 \\
\hline 3,4,5-Trimethyl- & & 1 & 0.039 & 126 & 7 & $T X-111$ \\
\hline E-2-Hexene & & 1 & 0.088 & 84 & 23 & BY-104 \\
\hline Z-2-Hexene & & 2 & 0.040 & 84 & 11 & BY-105 \\
\hline 4,4,5-Trimethyl- & & 1 & 0.034 & 126 & 6 & U-106 \\
\hline \multicolumn{7}{|l|}{ Z-3-Hexene } \\
\hline 2,3,4,5-Tetramethyl- & & 2 & 0.079 & 140 & 13 & $C-110$ \\
\hline 3-Ethyl-2,5-dimethyl- & $(*)$ & 2 & 0.280 & 140 & 45 & BY -107 \\
\hline 1-Heptene & & 17 & 1.700 & 98 & 389 & BY -108 \\
\hline 3-Methyl- & & 2 & 0.780 & 112 & 156 & BY-108 \\
\hline 5-Methyl- & & 2 & 0.110 & 112 & 22 & BY-109 \\
\hline 6-Methyl- & & 2 & 0.740 & 112 & 148 & BY -107 \\
\hline 2,4-Dimethyl- & & 1 & 0.120 & 126 & 21 & BX-102 \\
\hline E-2-Heptene & & 7 & 0.032 & 98 & 7 & B-103 \\
\hline Z-2-Heptene & & 3 & 0.040 & 98 & 9 & BY-105 \\
\hline 3-Methyl- & $\left({ }^{*}\right)$ & 1 & 0.700 & 112 & 140 & TX-116 \\
\hline E-3-Heptene & $\left(^{*}\right)$ & 2 & 0.023 & 98 & 5 & BY-104 \\
\hline Z-3-Heptene & & 1 & 0.026 & 98 & 6 & B-103 \\
\hline 2,2,3,5,5,6,6-Heptamethyl- & & 2 & 0.220 & 196 & 25 & $C-101$ \\
\hline 1-Octene & & 7 & 0.920 & 112 & 184 & BY -108 \\
\hline 3-Ethyl- & & 1 & 0.190 & 140 & 30 & $S-103$ \\
\hline 2-Octene & $(* *)$ & 1 & 0.680 & 112 & 136 & $T X-116$ \\
\hline E-3-Octene & $(*)$ & 2 & 0.130 & 112 & 26 & BY-104 \\
\hline Z-3-Octene & $(*)$ & 1 & 0.390 & 112 & 78 & BY-108 \\
\hline 1-Nonene & & 1 & 0.460 & 126 & 82 & BY-108 \\
\hline \multicolumn{7}{|l|}{ 4-Nonene } \\
\hline \multirow{2}{*}{\multicolumn{7}{|c|}{ Z-4-Nonene }} \\
\hline & & & & & & \\
\hline 2,3,3-Trimethyl- & & 3 & 0.570 & 168 & 76 & BY-108 \\
\hline 1-Decene & & 5 & 0.044 & 140 & 7 & U-103 \\
\hline E-2-Decene & & 1 & 0.043 & 140 & 7 & $C-102$ \\
\hline \multicolumn{7}{|l|}{ Z-2-Decene } \\
\hline 4-Methyl- & & 2 & 0.660 & 154 & 96 & $C-102$ \\
\hline 3-Decene & $(* *)$ & 2 & 0.150 & 140 & 24 & $C-101$ \\
\hline
\end{tabular}


Table 3a. Alkenes and Alkadienes (Page 2 of 3)

\begin{tabular}{|c|c|c|c|c|c|c|}
\hline Compound & & $\begin{array}{c}\text { Total Number } \\
\text { of } \\
\text { Observations }\end{array}$ & $\begin{array}{l}\text { Maximum } \\
\text { Concentration, } \\
\mathrm{mg} / \mathrm{m}^{3}\end{array}$ & MW & $\begin{array}{c}\text { Maximum } \\
\text { Concentration, } \\
\text { ppbv }\end{array}$ & $\begin{array}{l}\text { Tank with } \\
\text { Maximum } \\
\text { Concentration }\end{array}$ \\
\hline 3-Methy|- & & $\overline{1}$ & 0.190 & $\overline{154}$ & $\overline{28}$ & BY-107 \\
\hline 5-Decene & & 1 & 0.740 & 140 & 118 & BY-108 \\
\hline E-5-Decene & & 2 & 0.023 & 140 & 4 & B-103 \\
\hline 1-Undecene & & 4 & 0.320 & 154 & 47 & BY -108 \\
\hline 4-Methyl- & & 3 & 1.500 & 168 & 200 & BY-108 \\
\hline 7-Methyl- & & 2 & 0.080 & 168 & 11 & $C-110$ \\
\hline 8-Methyi- & & 1 & 0.050 & 168 & 7 & $C-110$ \\
\hline \multicolumn{7}{|l|}{ E-2-Undecene } \\
\hline 6-Methyl- & & 1 & 0.180 & 168 & 24 & BY-107 \\
\hline 7-Methyl- & & 1 & 0.082 & 168 & 11 & BY -107 \\
\hline 2,5-Dimethyl- & & 1 & 0.091 & 182 & 11 & U-106 \\
\hline \multicolumn{7}{|l|}{ Z-2-Undecene } \\
\hline 8-Methyl- & $(* *)$ & 1 & 0.380 & 168 & 51 & BY -108 \\
\hline 2,5-Dimethyl- & & 1 & 0.091 & 182 & 11 & BY-107 \\
\hline E-3-Undecene & & 2 & 0.023 & 154 & 3 & $T-111$ \\
\hline Z-3-Undecene & $\left({ }^{* *}\right)$ & & & & & \\
\hline 2-Methyl- & & 2 & 3.300 & 168 & 440 & C-102 \\
\hline \multicolumn{7}{|l|}{ 4-Undecene } \\
\hline 4-Methyl- & $(*)$ & 2 & 2.000 & 168 & 267 & $C-102$ \\
\hline 6-Methyl- & $(* *)$ & 2 & 0.240 & 168 & 32 & $B X-104$ \\
\hline \multicolumn{7}{|l|}{ Z-4-Undecene } \\
\hline 5-Methyl- & & 1 & 0.270 & 168 & 36 & C-102 \\
\hline E-5-Undecene & $(*)$ & 6 & 1.500 & 154 & 218 & $B Y-108$ \\
\hline 7-Methyl- & $(*)$ & 1 & 0.740 & 168 & 99 & C-102 \\
\hline Z-5-Undecene & & 6 & 1.500 & 154 & 218 & BY-108 \\
\hline 7-Methyl- & & 1 & 0.110 & 168 & 15 & BY -107 \\
\hline 1-Dodecene & & 6 & 0.430 & 168 & 57 & C-102 \\
\hline E-3-Dodecene & & 4 & 0.170 & 168 & 23 & TY-103 \\
\hline Z-3-Dodecene & & 1 & 0.300 & 168 & 40 & BY -108 \\
\hline E-4-Dodecene & & 3 & 0.610 & 168 & 81 & C-102 \\
\hline 1-Tridecene & & 7 & 0.100 & 182 & 12 & $C-110$ \\
\hline 6-Tridecene & $(* *)$ & 3 & 0.110 & 182 & 14 & $T Y-103$ \\
\hline 7-Methyl- & $(* *)$ & 5 & 2.300 & 196 & 263 & BY -108 \\
\hline 1-Tetradecene & & 4 & 5.700 & 196 & 651 & $C-103$ \\
\hline E-3-Tetradecene & & 3 & 0.710 & 196 & 81 & BY-107 \\
\hline Z-3-Tetradecene & & 1 & 0.250 & 196 & 29 & C-110 \\
\hline E-5-Tetradecene & & 2 & 0.630 & 196 & 72 & BY-108 \\
\hline Z-6-Tetradecene & & 2 & 0.040 & 196 & 5 & BY-105 \\
\hline Z-7-Tetradecene & $(*)$ & 4 & 0.019 & 196 & 2 & $T-111$ \\
\hline 1-Pentadecene & & 4 & 0.330 & 210 & 35 & BY-107 \\
\hline 1-Hexadecene & & 6 & 2.600 & 224 & 260 & C-103 \\
\hline Z-3-Hexadecene & & 2 & 0.040 & 224 & 4 & BY-104 \\
\hline Z-7-Hexadecene & & 3 & 0.220 & 224 & 22 & BY -108 \\
\hline E-5-Eicosene & & 1 & 0.680 & 294 & 52 & BY-108 \\
\hline \multicolumn{7}{|l|}{ ALKADIENES } \\
\hline 1,3-Butadiene & & 17 & 0.560 & 54 & 232 & $C-204$ \\
\hline 2-Methyl- & & 3 & 0.160 & 68 & 53 & BY-104 \\
\hline 2,3-Dimethyl- & & 1 & 0.048 & 82 & 13 & BY-106 \\
\hline 1,2-Pentadiene & & 1 & 0.033 & 68 & 11 & BY-105 \\
\hline E-1,3-Pentadiene & & 1 & 0.027 & 68 & 9 & BY-106 \\
\hline Z-1,3-Pentadiene & & 1 & 0.084 & 68 & 28 & $B Y-110$ \\
\hline 2-Methyl- & $\left({ }^{*}\right)$ & 4 & 1.500 & 82 & 410 & BY-108 \\
\hline 1,4-Hexadiene & $(* *)$ & 5 & 0.160 & 82 & 44 & BY-104 \\
\hline 4-Methyl- & $(\star \star)$ & 2 & 0.030 & 96 & 7 & BY-104 \\
\hline 1,5-Hexadiene & & 1 & 0.120 & 68 & 40 & BY-104 \\
\hline 2,6-Octadiene & $(* *)$ & & & & & \\
\hline 4,5-Dimethyl- & $(* *)$ & 1 & 0.028 & 138 & 5 & BY-109 \\
\hline 3,4-Nonadiene & & 1 & 0.480 & 124 & 87 & BY -108 \\
\hline \multirow{2}{*}{\multicolumn{7}{|c|}{$\begin{array}{l}\text { 4,b-Nonadiene } \\
\text { E,E-4,6-Decadiene }\end{array}$}} \\
\hline & & & & & & \\
\hline 3,8-Dimethyl- & & 1 & 0.470 & 166 & 63 & BY-106 \\
\hline
\end{tabular}

POLYENES 
Table 3a. Alkenes and Alkadienes (Page 3 of 3)

Compound

2,6,10,14,18,22-Tetracosahexaene

$2,6,10,19,23-P e n t a m e t h y l-$

$2,6,10,15,19,23$-Hexamethyl-

Total
Total Number

of

Observations

Observations

2

513

3

Maximum

Concentration, $\mathrm{mg} / \mathrm{m}^{3}$

1.100

1.200

131.0
Maximum

Concentration,

ppbv

406

420
MW

Notes

$\left({ }^{*}\right)$ The observations for unresolved mixtures have been included.

(**) The positional, geometrical or stereo isomer has not been defined. 
Table 3b. Alkenes and Alkadienes Observed in One Tank at Concentration Less Than $0.025 \mathrm{mg} / \mathrm{m}^{3}$

\begin{tabular}{|c|c|c|c|c|c|c|c|}
\hline Compound & & $\begin{array}{c}\text { Total Number } \\
\text { of } \\
\text { Observations }\end{array}$ & $\begin{array}{c}\text { Maximum } \\
\text { Concentration, } \\
\mathrm{mg} / \mathrm{m}^{3}\end{array}$ & MW & $\begin{array}{c}\text { Maximum } \\
\text { Concentration, } \\
\text { ppby }\end{array}$ & $\begin{array}{c}\text { Tank with } \\
\text { Maximum } \\
\text { Concentration } \\
\end{array}$ & $\begin{array}{l}\text { TWINS2 } \\
\text { (CAS) } \\
\text { Number }\end{array}$ \\
\hline \multicolumn{8}{|l|}{ ALKENES } \\
\hline 1-Butene & & & & & & & \\
\hline 2,3-Dimethyl- & & 1 & 0.0250 & 84 & 7 & BY-105 & $563-78-0$ \\
\hline \multicolumn{8}{|l|}{ 1-Pentene } \\
\hline 2,2,4-Trimethyl- & & 1 & 0.0190 & 112 & 4 & $T-111$ & $107-39-1$ \\
\hline \multicolumn{8}{|l|}{ 2-Pentene } \\
\hline 3-Ethy!- & & 1 & 0.0100 & 98 & 2 & $T Y-104$ & $816-79-5$ \\
\hline \multicolumn{8}{|l|}{ 1-Hexene } \\
\hline 2-Methyl- & & 1 & 0.0040 & 98 & 1 & BY-106 & $6094-02-6$ \\
\hline 3,5,5-Trimethyl- & & 1 & 0.0100 & 126 & 2 & BY-105 & $4316-65-8$ \\
\hline \multicolumn{8}{|l|}{ 2-Hexene } \\
\hline 2,4-Dimethyl- & & $i$ & 0.0090 & 112 & 2 & C-105 & $14255-23-3$ \\
\hline \multicolumn{8}{|l|}{ 3-Heptene } \\
\hline 4-Propyl- & $(*)$ & 1 & 0.0130 & 140 & 2 & B-103 & $4485-13-6$ \\
\hline \multicolumn{8}{|l|}{ 1-Octene } \\
\hline 7-Methyl- & & 1 & 0.0220 & 126 & 4 & C-104 & $13151-06-9$ \\
\hline E-4-Octene & & 1 & 0.0100 & 112 & 2 & BY-105 & $14850-23-8$ \\
\hline E-2-Nonene & & 1 & 0.0007 & 126 & 0.1 & $T Y-104$ & $6434-78-2$ \\
\hline \multicolumn{8}{|l|}{ 4-Nonene } \\
\hline 5-Methyl- & & 1 & 0.0030 & 140 & 0.5 & BY-106 & $15918-07-7$ \\
\hline \multicolumn{8}{|l|}{ 1-Decene } \\
\hline 4-Methyl- & & 1 & 0.0080 & 154 & 1 & TY-104 & $13151-29-6$ \\
\hline 5-Methyl- & & 1 & 0.0019 & 154 & 0.3 & $T Y-104$ & $54244-79-0$ \\
\hline 3,4-Dimethyl- & & 1 & 0.0130 & 168 & 2 & TY-104 & \\
\hline \multicolumn{8}{|l|}{ 4-Decene } \\
\hline 7-Methyl- & & 1 & 0.0070 & 154 & 1 & TY-104 & UAE011-05 \\
\hline \multicolumn{8}{|l|}{ 2-Undecene } \\
\hline 4-Methyl- & $(*)$ & 1 & 0.0035 & 168 & 0.5 & $T Y-104$ & $91695-32-8$ \\
\hline \multicolumn{8}{|l|}{ E-2-Undecene } \\
\hline 4,5-Dimethyl- & & 1 & 0.0011 & 182 & 0.1 & U-106 & $55170-92-8$ \\
\hline Z-2-Undecene & & 1 & 0.0006 & 154 & 0.1 & $T Y-104$ & $821-96-5$ \\
\hline \multicolumn{8}{|l|}{ 3-Undecene } \\
\hline 5-Methyl- & $(*)$ & 1 & 0.0080 & 168 & 1 & TY-104 & UAE012-06 \\
\hline 8-Methyl- & $(*)$ & 1 & 0.0100 & 168 & 1 & BY-105 & UAE012-04 \\
\hline E-4-Undecene & & 1 & 0.0040 & 154 & 1 & C-107 & $693-62-9$ \\
\hline \multicolumn{8}{|l|}{ 2-Dodecene } \\
\hline 4-Methyl- & (*) & 1 & 0.0022 & 182 & 0.3 & $C-110$ & $56851-45-7$ \\
\hline Z-5-Dodecene & 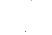 & 1 & 0.0073 & 168 & 1 & BY-106 & $7206-28-2$ \\
\hline E-6-Dodecene & & 1 & 0.0100 & 168 & 1 & BY -105 & $7206-17-9$ \\
\hline 1-Heptadecene & & 1 & 0.0078 & 238 & 1 & $5 X-103$ & $6765-39-5$ \\
\hline 1-Octadecene & & 1. & 0.0020 & 252 & 0.2 & $C-111$ & $112-88-9$ \\
\hline 1-Nonadecene & & 1 & 0.0010 & 266 & 0.1 & $C-109$ & $18435-45-5$ \\
\hline \multicolumn{8}{|l|}{ ALKADIENES } \\
\hline \multicolumn{8}{|l|}{ 1,3-Pentadiene } \\
\hline 2,3-Dimethyl- & & 1 & 0.0110 & 96 & 3 & TY-104 & $1113-56-0$ \\
\hline 1,4-Pentadiene & & & & & & & \\
\hline $\begin{array}{l}\text { 2,3-Dimethyl- } \\
\text { Z-1,3-Hexadiene }\end{array}$ & & 1 & 0.0075 & 96 & 2 & SX-106 & $758-86-1$ \\
\hline 3-Ethyl-2-methyl- & & 1 & 0.0052 & 124 & 1 & $A X-101$ & $74752-97-9$ \\
\hline 1,4-Hexadiene & & & & & & & \\
\hline 2,3-Dimethyl- & $\left({ }^{*}\right)$ & 1 & 0.0050 & 110 & 1 & $A X-101$ & $18669-52-8$ \\
\hline 1,11-Dodecadiene & & 1 & 0.0040 & 166 & 1 & $T Y=104$ & $5876-87-9$ \\
\hline 1,12-Tridecadiene & & 1 & 0.0050 & 180 & 1 & C-105 & $21964-48-7$ \\
\hline Total & & 33 & 0.2508 & & 45 & & \\
\hline
\end{tabular}

NOTES

()) The positional, geometrical or stereo isomer has not been defined. 
Table 4a. Alkynes

\begin{tabular}{|c|c|c|c|c|c|}
\hline Compound & $\begin{array}{c}\begin{array}{c}\text { Total Number } \\
\text { of }\end{array} \\
\text { Observations } \\
\end{array}$ & $\begin{array}{c}\text { Maximum } \\
\text { Concentration, } \\
\mathrm{mg} / \mathrm{m}^{3} \\
\end{array}$ & MW & $\begin{array}{c}\begin{array}{c}\text { Maximum } \\
\text { Concentration, } \\
\text { ppbv }\end{array} \\
\end{array}$ & $\begin{array}{c}\text { Tank with } \\
\text { Maximum } \\
\text { Concentration }\end{array}$ \\
\hline Propyne & 4 & 0.610 & $\overline{40}$ & 342 & $C-102$ \\
\hline 4-Nonyne & 1 & 0.610 & 124 & 110 & BY -108 \\
\hline 3-Decyne & 2 & 0.510 & 138 & 83 & BY -108 \\
\hline 3-Hexadecyne & 1 & 0.500 & 222 & 50 & C-102 \\
\hline Total & 8 & 2.230 & & 585 & \\
\hline
\end{tabular}


Table 4b. Alkynes Observed in One Tank at Concentration Less Than $0.025 \mathrm{mg} / \mathrm{m}^{3}$

\begin{tabular}{|c|c|c|c|c|c|c|}
\hline Compound & $\begin{array}{c}\text { Total Number } \\
\text { of } \\
\text { Observations } \\
\end{array}$ & $\begin{array}{c}\text { Maximum } \\
\text { Concentration, } \\
\mathrm{mg} / \mathrm{m}^{3} \\
\end{array}$ & MW & $\begin{array}{c}\text { Maximum } \\
\text { Concentration, } \\
\text { ppbv } \\
\end{array}$ & $\begin{array}{c}\text { Tank with } \\
\text { Maximum } \\
\text { Concentration } \\
\end{array}$ & $\begin{array}{l}\text { TWINS2 } \\
\text { (CAS) } \\
\text { Number } \\
\end{array}$ \\
\hline 1-Nonyne & 1 & 0.010 & $\overline{124}$ & $\overline{2}$ & $B Y-105$ & $3452-09-3$ \\
\hline 1-Octadecyne & 1 & 0.002 & 252 & 0.2 & $T-107$ & $629-89-0$ \\
\hline Total & 2 & 0.012 & & 2 & & \\
\hline
\end{tabular}


Table 5a. Benzene, Biphenyl, Napthalene, Fluorene and Their Alkyl and Alkenyl Derivatives

\begin{tabular}{|c|c|c|c|c|c|c|}
\hline Compound & \multicolumn{2}{|c|}{$\begin{array}{c}\text { Total Number } \\
\text { of } \\
\text { Observations }\end{array}$} & \multirow[t]{2}{*}{$\begin{array}{c}\text { Maximum } \\
\text { Concentration, } \\
\mathrm{mg} / \mathrm{m}^{3} \\
\end{array}$} & \multirow[t]{2}{*}{ MW } & \multirow[t]{2}{*}{$\begin{array}{c}\text { Maximum } \\
\text { Concentration, } \\
\text { ppbv } \\
\end{array}$} & \multirow[t]{2}{*}{$\begin{array}{c}\text { Tank with } \\
\text { Maximum } \\
\text { Concentration } \\
\end{array}$} \\
\hline$\overline{\text { BENZENE AND ALKYLBENZE }}$ & NES & & & & & \\
\hline Benzene & & 175 & 7.500 & 78 & 2154 & $B Y-104$ \\
\hline \multicolumn{7}{|l|}{ Monosubstituted Benzenes } \\
\hline Methyl- & & 208 & 4.800 & 92 & 1169 & $\mathrm{BX}-103$ \\
\hline Ethyl- & & 110 & 0.660 & 106 & 139 & $B X-103$ \\
\hline (1-Methylethyl)- & & 2 & 0.470 & 120 & 88 & C-102 \\
\hline Propyl- & & 4 & 0.028 & 120 & 5 & $C-110$ \\
\hline (1,1-Dimethylethyl)- & & 2 & 0.053 & 134 & 9 & C-110 \\
\hline Pentyl- & & 2 & 1.400 & 148 & 212 & $C-102$ \\
\hline (1-Methylpentyl)- & & 1 & 0.890 & 162 & 123 & C-102 \\
\hline (1-Methylhexyl)- & & 1 & 0.720 & 176 & 92 & C-102 \\
\hline (1-Methylheptyl)- & & 1 & 0.180 & 190 & 21 & $C-102$ \\
\hline (1-Methyldecyl)- & & 2 & 0.006 & 232 & 1 & C-104 \\
\hline (1-Propylnonyl)- & & 2 & 0.005 & 246 & 0.5 & C-104 \\
\hline (1-Butylheptyl)- & $\cdot$ & 2 & 0.005 & 232 & 0.5 & $C-104$ \\
\hline (1-Pentylheptyl)- & & 2 & 0.007 & 246 & 1 & C-104 \\
\hline \multicolumn{7}{|l|}{ Disubstituted Benzenes } \\
\hline 1,2-Dimethyl- & & 98 & 0.780 & 106 & 165 & $B Y-108$ \\
\hline 1,3- and 1,4-Dimethyl- & & 128 & 1.800 & 106 & 380 & $B X-103$ \\
\hline 1-Ethyl-2-methyl- & 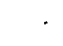 & 40 & 0.044 & 120 & 8 & $\mathrm{C}-110$ \\
\hline 1-Ethyl-3-methyl- & & 1 & 0.036 & 120 & 7 & $5 X-107$ \\
\hline 1-Ethyl-3-methyl- & & 4 & 0.011 & 120 & 2 & BY-104 \\
\hline 1-Methyl-2-propyl- & & 1 & 0.870 & 134 & 145 & $\mathrm{C}-102$ \\
\hline \multicolumn{7}{|l|}{ Trisubstituted Benzenes } \\
\hline 1,2,4-Trimethyl- & & 63 & 0.078 & 120 & 15 & C-107 \\
\hline 1,3,5-Trimethyl- & & 48 & 0.052 & 120 & 10 & $\mathrm{U}-112$ \\
\hline \multicolumn{7}{|l|}{ BIPHENYL } \\
\hline 1,1'-Biphenyl & & 6 & 15.000 & 154 & 2182 & $C-103$ \\
\hline 2-Methyl- & & 3 & 1.800 & 168 & 240 & C-102 \\
\hline 2,2'-Diethyl- & & 4 & 0.003 & 210 & 0.3 & $T X-105$ \\
\hline \multicolumn{7}{|l|}{ OTHER ARENES } \\
\hline Naphthalene & & 8 & 0.045 & 128 & 8 & $s x-112$ \\
\hline 2-Methyl- & & 1 & 0.027 & 142 & 4 & $T-111$ \\
\hline$(9 \mathrm{H})$ Fluorene & & 2 & 0.017 & 166 & 2 & $C-102$ \\
\hline \multirow{2}{*}{\multicolumn{7}{|c|}{$\begin{array}{l}\text { ALKENYLBENZENES } \\
\text { Benzene }\end{array}$}} \\
\hline & & & & & & \\
\hline 1-Ethenyl- & & 48 & 1.300 & 104 & 280 & $C-103$ \\
\hline (1-Methylethenyl)- & & 2 & 0.014 & 118 & 3 & $C-108$ \\
\hline (1-Propenyl)- & $\left({ }^{\star}\right)$ & 2 & 0.630 & 118 & 120 & C- 102 \\
\hline (2-Propenyl)- & & 2 & 0.290 & 118 & 55 & $C-101$ \\
\hline (2-Methyl-2-propenyl)- & & 1 & 0.590 & 132 & 100 & C-102 \\
\hline Total & & 976 & 40.111 & & 7740 & \\
\hline
\end{tabular}

Notes

$\left.{ }^{\star}\right)$ The positional, geometrical or stereo isomer has not been defined. 
Table 5b. Benzene, Biphenyl, Napthalene, Fluorene and Their Alkyl and Alkenyl Derivatives Observed in One Tank at Concentrations Less Than $0.025 \mathrm{mg} / \mathrm{m}^{3}$

\begin{tabular}{|c|c|c|c|c|c|c|}
\hline Compound & $\begin{array}{c}\text { Total Number } \\
\text { of } \\
\text { Observations }\end{array}$ & $\begin{array}{c}\text { Maximum } \\
\text { Concentration, } \\
\mathrm{mg} / \mathrm{m}^{3}\end{array}$ & MW & $\begin{array}{c}\text { Maximum } \\
\text { Concentration, } \\
\text { ppbv } \\
\end{array}$ & $\begin{array}{c}\text { Tank with } \\
\text { Maximum } \\
\text { Concentration }\end{array}$ & $\begin{array}{c}\text { TWINS2 } \\
\text { (CAS) } \\
\text { Number } \\
\end{array}$ \\
\hline \multicolumn{7}{|l|}{ Monosubstituted Benzenes } \\
\hline (1-MethylpropyI)- & 1 & 0.0200 & 134 & 3 & $T-111$ & $135-98-8$ \\
\hline (1,1-Dimethylpropy) & 1 & 0.0190 & 148 & 3 & $T-111$ & 2049-95-8 \\
\hline (1-Ethyinonyl)- & $i$ & 0.0080 & 218 & 1 & C.105 & $4536-87-2$ \\
\hline (1-Butyioctyi)- & 1 & 0.0060 & 246 & 1 & C-105 & $2719-63-3$ \\
\hline (1-Methylundecyi)- & 1 & 0.0050 & 246 & 0.5 & C-104 & $2719-61-1$ \\
\hline \multicolumn{7}{|l|}{ Disubstituted Benzenes } \\
\hline 1-Methyl-3-(1-methylethyl)- & 1 & 0.0140 & 134 & 2 & $T-111$ & $535-77-3$ \\
\hline 1-Isopropyl-2-methyl- & 1 & 0.0200 & 134 & 3 & SX-107 & $527-84-4$ \\
\hline \multicolumn{7}{|l|}{ Trisubstituted Benzenes } \\
\hline 1,2,3-Trimethyl- & 1 & 0.0240 & 120 & 4 & $5 \times-107$ & $526-73-8$ \\
\hline bis-1,1'-(1,4-Butanediy $)$ benzene & 1 & 0.0130 & 210 & 1 & $T X-105$ & $1083-56-3$ \\
\hline Naphthalene & & & & & & \\
\hline 1-Methyl- & 1 & 0.0240 & 142 & 4 & $S \times-107$ & $90-12-0$ \\
\hline 1,7-Dimethyl- & 1 & 0.0110 & 156 & 2 & $T-111$ & $575-37-1$ \\
\hline 2,3-Dimethyl- & 1 & 0.0120 & 156 & 2 & $T-111$ & $581-40-8$ \\
\hline Total & 12 & 0.1760 & & 27 & & \\
\hline
\end{tabular}


Table 6a. Halogen Containing Compounds (Page 1 of 1)

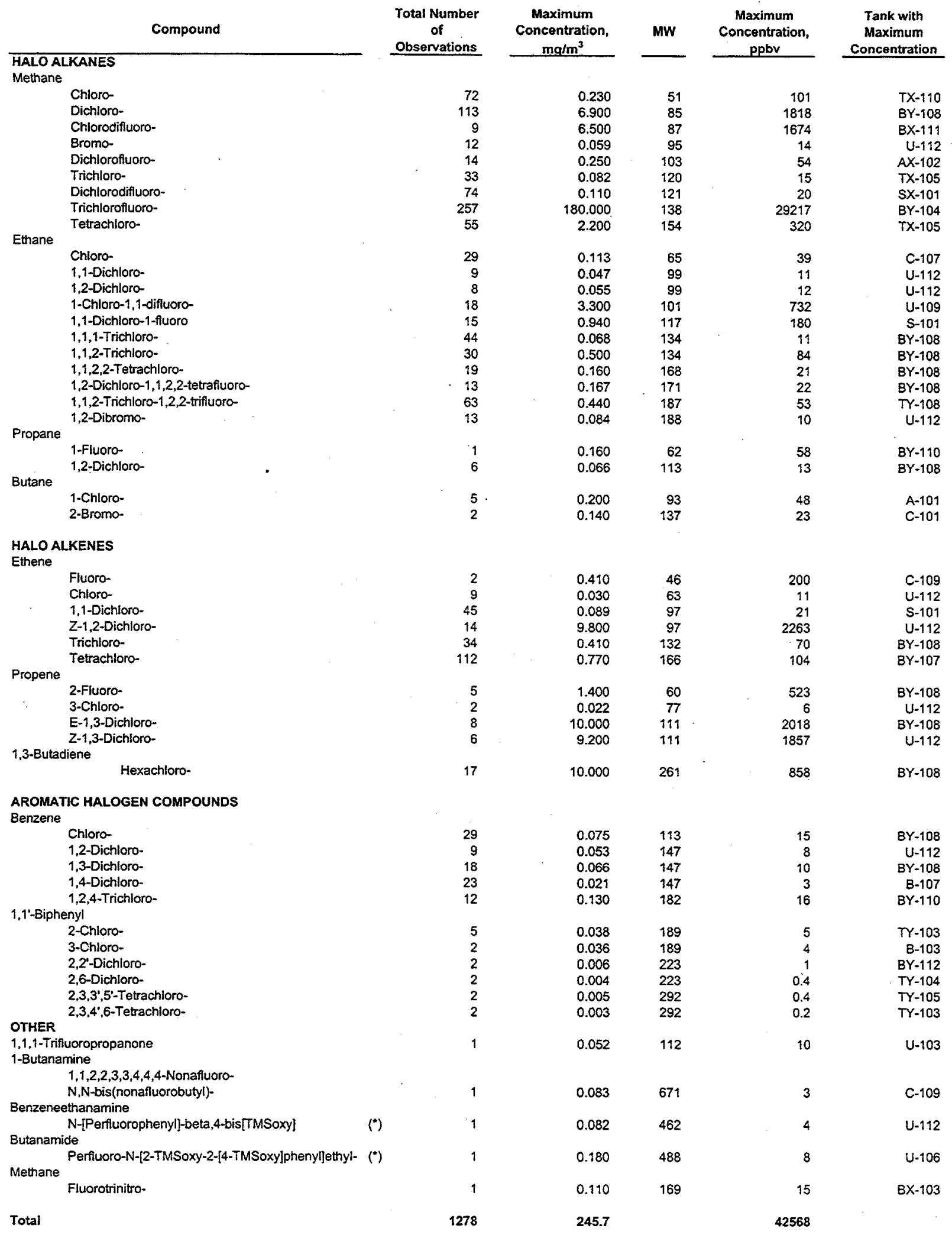

Note

(*) Hexachloro-1,3-butadiene, listed in the first version of this report, has been removed. All positive identifications of this compound have been determined to be suspect, and have been so incicated in the Tank Characterization Database.

("*) The trimethylsilyl fragment is designated as TMS in this table. 
Table 6b. Halogen Containing Compounds Observed in One Tank at Concentration Less Than $0.025 \mathrm{mg} / \mathrm{m}^{3}$

\begin{tabular}{|c|c|c|c|c|c|c|}
\hline Compound & $\begin{array}{c}\text { Total Number } \\
\text { of } \\
\text { Observations }\end{array}$ & $\begin{array}{c}\text { Maximum } \\
\text { Concentration, } \\
\mathrm{mg} / \mathrm{m}^{3}\end{array}$ & MW & $\begin{array}{c}\text { Maximum } \\
\text { Concentration, } \\
\text { ppbv }\end{array}$ & $\begin{array}{c}\text { Tank with } \\
\text { Maximum } \\
\text { Concentration } \\
\end{array}$ & $\begin{array}{c}\text { TWINS2 } \\
\text { (CAS) } \\
\text { Number }\end{array}$ \\
\hline \multicolumn{7}{|l|}{$\begin{array}{l}\text { HALOALKANES } \\
\text { Propane }\end{array}$} \\
\hline Octane 1 1-Chloro-2,2-dimethyl- & 1 & 0.0180 & 107 & 4 & U-105 & $753-89-9$ \\
\hline 2-Chiloro- & 1 & 0.0047 & 149 & 1 & $T X-118$ & $628-61-5$ \\
\hline \multicolumn{7}{|c|}{$\begin{array}{l}\text { AROMATIC HALOGEN COMPOUNDS } \\
\text { Benzene }\end{array}$} \\
\hline $\begin{array}{l}\text { 1-Chloro-4-nitro- } \\
\text { Biphenyl }\end{array}$ & 1 & 0.0053 & 134 & 1 & $C-107$ & $100-00-5$ \\
\hline $\begin{array}{l}\text { 4-Chloro- } \\
\text { 3-Dichloro- }\end{array}$ & 1 & $\begin{array}{l}0.0028 \\
0.0011\end{array}$ & $\begin{array}{l}189 \\
223\end{array}$ & $\begin{array}{l}0.3 \\
0.1\end{array}$ & $\begin{array}{l}T Y-104 \\
T Y-104\end{array}$ & $\begin{array}{r}2051-62-9 \\
16605-91-7\end{array}$ \\
\hline 2,5-Dichloro- & 1 & 0.0010 & 223 & 0.1 & TY-104 & $34883-39-1$ \\
\hline 3,3'-Dichloro- & 1 & 0.0075 & 223 & 1 & $T X-118$ & $2050-67-1$ \\
\hline 4,4'-Dichloro- & 1 & 0.0041 & 223 & 0.4 & $T Y-104$ & $2050-68-2$ \\
\hline $2,2,4^{\circ}$-Trichloro- & 1 & 0.0026 & 257 & 0.2 & TY-104 & $7012-37-5$ \\
\hline 2,4,5-Trichloro- & 1 & 0.0038 & 257 & 0.3 & $T Y-104$ & $16606-02-3$ \\
\hline $2,3,4$-Trichloro- & 1 & 0.0019 & 257 & 0.2 & TY-104 & $55702-46-0$ \\
\hline \multicolumn{7}{|l|}{$\begin{array}{l}\text { OTHER HALOGEN COMPOUNDS } \\
\text { Silane }\end{array}$} \\
\hline Fluorotrimethyl- & 1 & 0.0020 & 92 & 0.5 & $T-107$ & $420-56-4$ \\
\hline Total & 12 & 0.05480 & & 8.279 & & \\
\hline
\end{tabular}


Table 7a. Alcohols, Phenols, and Ethers (Page 1 of 2)

\begin{tabular}{|c|c|c|c|c|c|}
\hline Compound & $\begin{array}{c}\text { Total Number } \\
\text { of } \\
\text { Observations }\end{array}$ & $\begin{array}{c}\text { Maximum } \\
\text { Concentration, } \\
\mathrm{mg} / \mathrm{m}^{3}\end{array}$ & MW & $\begin{array}{c}\text { Maximum } \\
\text { Concentration, } \\
\text { ppbv }\end{array}$ & $\begin{array}{c}\text { Tank with } \\
\text { Maximum } \\
\text { Concentration }\end{array}$ \\
\hline \multicolumn{6}{|l|}{$\overline{\text { ALKANOLS }}$} \\
\hline Methanol & 155 & 30.000 & 32 & 21000 & S-102 \\
\hline Ethanol & 172 & 44.000 & 46 & 21426 & S-102 \\
\hline 2-Butoxy- & 11 & 0.320 & 118 & 61 & BY-104 \\
\hline 2-Tetradecyloxy- & 1 & 2.100 & 258 & 182 & BY-108 \\
\hline 1-Propanol & 171 & 4.700 & 60 & 1755 & BY-107 \\
\hline 2-Methyl- & 6 & 0.060 & 74 & 18 & $T Y-104$ \\
\hline 2,2-Dimethyl- & 7 & 7.800 & 88 & 1985 & C-103 \\
\hline 1-Cyclopentyl-2,2-dimethyl- & 2 & 0.012 & 156 & 2 & U-112 \\
\hline 2-Propanol & 65 & 5.300 & 60 & 1979 & BY-108 \\
\hline 1-(1-Methylethoxy)- & 1 & 0.033 & 118 & 6 & U-107 \\
\hline 2-Methyl- & 30 & 0.330 & 74 & 100 & $T X-110$ \\
\hline 2-Propyl- & 77 & 5.300 & 102 & 1164 & $T X-110$ \\
\hline 1-Butanol & 222 & 193.000 & 74 & 58422 & BY-108 \\
\hline 2-Methyl- & 4 & 0.160 & 88 & 41 & C-101 \\
\hline 3-Methyl- & 2 & 0.110 & 88 & 28 & BX-104 \\
\hline 2-Ethyl- & 2 & 0.064 & 102 & 14 & $T Y-103$ \\
\hline 3,3-Dimethyl- & 3 & 0.083 & 102 & 18 & $T X-111$ \\
\hline 2-Butanol & 24 & 0.680 & 74 & 206 & BY-107 \\
\hline 2-Methyl- & 3 & 0.070 & 88 & 18 & BY-104 \\
\hline 1-Pentanol & 17 & 0.190 & 88 & 48 & BX-104 \\
\hline 4-Methyl-2-propyl- & 1 & 0.035 & 144 & 5 & TY-103 \\
\hline 2-Pentanol & 3 & 0.550 & 88 & 140 & BY-107 \\
\hline 2-Methyl- & 6 & 0.230 & 102 & 51 & BY-107 \\
\hline 2,3-Dimethyl- & 2 & 0.006 & 116 & 1 & BY-106 \\
\hline 3-Ethyl-2-methyl- & 2 & 0.001 & 130 & 0.2 & $T-107$ \\
\hline \multicolumn{6}{|l|}{ 3-Pentanol } \\
\hline 2-Methyi- & 3 & 0.150 & 102 & 33 & BY-108 \\
\hline 1-Hexanol & 16 & 0.210 & 102 & 46 & $B X-103$ \\
\hline 2-Ethyl- & 44 & 1.600 & 130 & 276 & C-103 \\
\hline 3-Hexanol & 1 & 0.037 & 102 & 8 & U-106 \\
\hline 2-Methyl- & 1 & 0.093 & 116 & 18 & BY -102 \\
\hline 1-Heptanol & 15 & 2.100 & 116 & 406 & C-103 \\
\hline 2-Heptanol & 2 & 0.270 & 116 & 52 & BY-107 \\
\hline 2-Methyl- & 2 & 0.003 & 130 & 1 & BY-106 \\
\hline 3-Heptanol & 18 & 0.310 & 116 & 60 & BY-107 \\
\hline 1-Octanol & 16 & 0.360 & 130 & 62 & BY -108 \\
\hline 2-Butyl- & 5 & 0.350 & 186 & 42 & BY-108 \\
\hline 2-Octanol & 4 & 0.410 & 130 & 71 & BY -108 \\
\hline 2-Methyl- & 1 & 0.036 & 144 & 6 & BY-102 \\
\hline 1-Nonanol & 9 & 0.024 & 144 & 4 & BY -102 \\
\hline 1-Decanol & 2 & 0.002 & 158 & 0.3 & $C-109$ \\
\hline 1-Dodecanol & 3 & 0.008 & 186 & 1 & C-104 \\
\hline 6-Dodecanol & 2 & 0.014 & 186 & 2 & B-103 \\
\hline 1-Tetradecanol & 2 & 0.010 & 214 & 1 & BY-105 \\
\hline 1-Hexadecanol & 15 & 11.000 & 242 & 1018 & C-204 \\
\hline 1-Octadecanol & 4 & 12.000 & 270 & 996 & C-204 \\
\hline \multicolumn{6}{|l|}{ CYCLOALKANOLS } \\
\hline Cyclopentanol & 5 & 7.300 & 100 & 1635 & $C-103$ \\
\hline \multicolumn{6}{|l|}{ ALKANDIOLS } \\
\hline 1,2-Propandiol & 14 & 0.510 & 76 & 150 & A-101 \\
\hline \multicolumn{6}{|c|}{ ALKENOLS, ALKENDIOLS, AND ALKYNEOLS } \\
\hline 2-Propen-1-ol & 2 & 0.010 & 58 & 4 & $s x-106$ \\
\hline Z-2-Buten-1-ol & 2 & 0.120 & 72 & 37 & C-101 \\
\hline 3-Buten-1-ol & 2 & 18.000 & 72 & 5600 & BY-108 \\
\hline 3-Buten-2-ol & 7 & 4.800 & 72 & 1493 & BY -108 \\
\hline 1,4-Pentadien-3-ol & 1 & 0.027 & 84 & 7 & BY-110 \\
\hline 5-Hexen-1-ol & 1 & 0.071 & 100 & 16 & $A X-102$ \\
\hline
\end{tabular}


Table 7a. Alcohols, Phenols, and Ethers (Page 2 of 2)

\begin{tabular}{|c|c|c|c|c|c|}
\hline Compound & $\begin{array}{c}\text { Total Number } \\
\text { of } \\
\text { Observations }\end{array}$ & $\begin{array}{l}\text { Maximum } \\
\text { Concentration, } \\
\mathrm{mg} / \mathrm{m}^{3}\end{array}$ & MW & $\begin{array}{c}\text { Maximum } \\
\text { Concentration, } \\
\text { ppbv }\end{array}$ & $\begin{array}{c}\text { Tank with } \\
\text { Maximum } \\
\text { Concentration }\end{array}$ \\
\hline 2-Methyl- & $\overline{2}$ & 0.370 & 142 & 58 & $\mathrm{C}-101$ \\
\hline $\begin{array}{l}\text { 1-Dodecyn-4-ol } \\
6,10 \text {-Dodecadien-1-ol }\end{array}$ & 1 & 0.042 & 182 & 5 & BY -103 \\
\hline 3,7,11-Trimethyl- & 1 & 2.600 & 224 & 260 & C-103 \\
\hline \multicolumn{6}{|l|}{ ETHERS } \\
\hline $\begin{array}{l}\text { Dimethyl Ether } \\
\text { Butane }\end{array}$ & 11 & 4.200 & 46 & 2045 & C-103 \\
\hline 1-Methoxy- & 1 & 0.044 & 88 & 11 & $T X-111$ \\
\hline $\begin{array}{l}\text { Dibutyl Ether } \\
\text { E-2-Pentene }\end{array}$ & 8 & 2.300 & 130 & 396 & C-103 \\
\hline Hexane ${ }^{\text {5-Pentyloxy- }}$ & 2 & 0.024 & 156 & 3 & C-104 \\
\hline 3-Methoxy- & 2 & 0.240 & 116 & 46 & $B Y-107$ \\
\hline 1-Ethoxy- & 1 & 0.110 & 130 & 19 & $T Y-103$ \\
\hline \multicolumn{6}{|l|}{ Cycloheptane } \\
\hline Methoxy- & 2 & 0.110 & 128 & 19 & C-101 \\
\hline trans-1,3-Dimethoxy- & 1 & 0.002 & 158 & 0.3 & $T-107$ \\
\hline Didecyl ether & 4 & 0.970 & 298 & 73 & BY -108 \\
\hline Divinyl ether & 1 & 0.097 & 70 & 31 & C-107 \\
\hline Diphenyl Ether & 2 & 0.180 & 170 & 24 & $T-111$ \\
\hline \multicolumn{6}{|l|}{ PHENOLS } \\
\hline Phenol & 10 & 0.087 & 94 & 21 & BY -107 \\
\hline 2-Methyl- & 3 & 0.040 & 108 & 8 & BY -104 \\
\hline 3-Methyl- & 2 & 0.010 & 108 & 2 & BY-105 \\
\hline Total & 1242 & 366.4 & & 123,737 & \\
\hline
\end{tabular}


Table 7b. Alcohols, Phenols, and Ethers Observed in One Tank at Concentration Less Than $0.025 \mathrm{mg} / \mathrm{m}^{3}$ (Page 1 of 2)

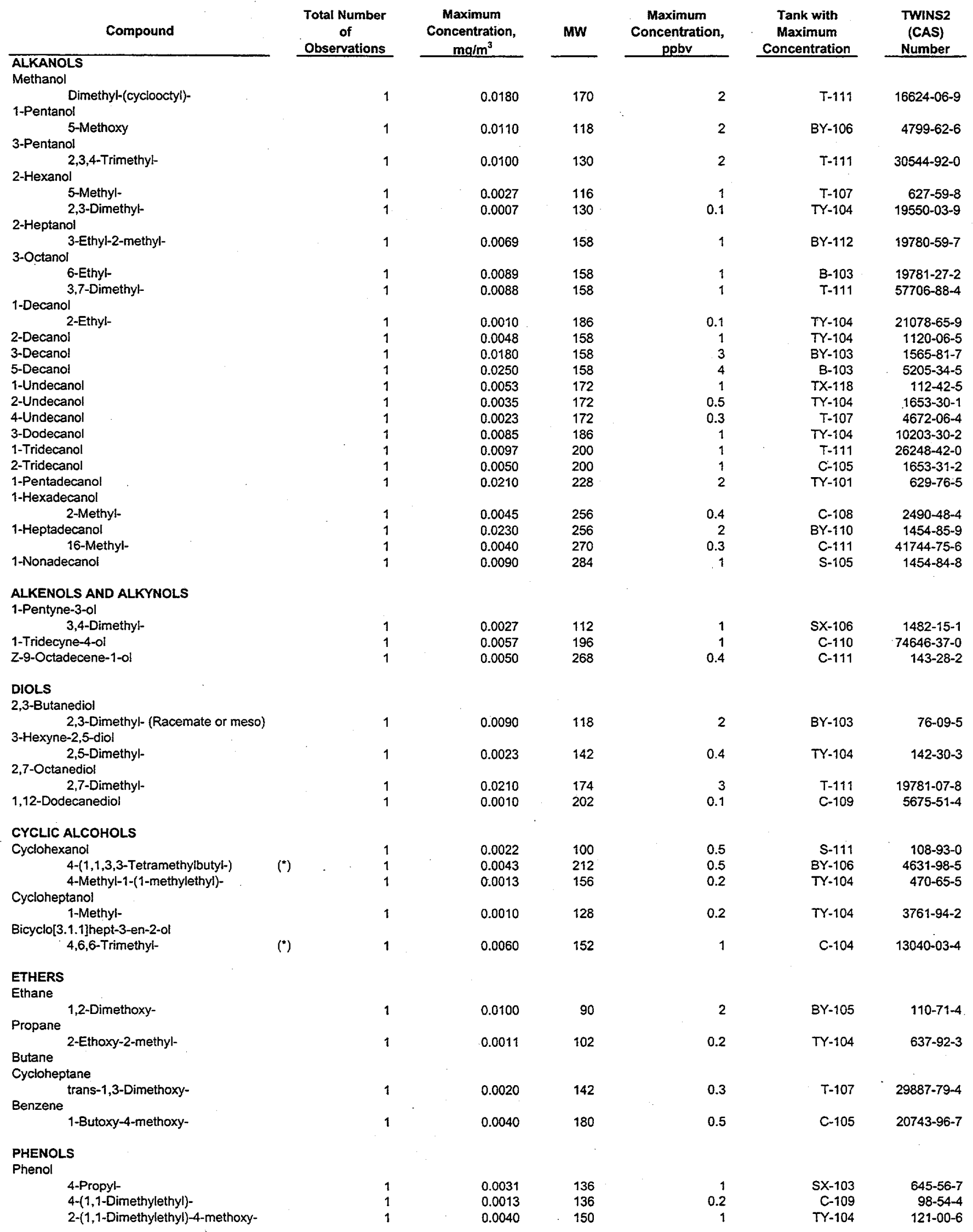


Table 7b. Alcohols, Phenols, and Ethers Observed in One Tank at Concentration Less Than $0.025 \mathrm{mg} / \mathrm{m}^{3}(\mathrm{Page} 2 \mathrm{of} 2)$

$\frac{\text { Compound }}{1,3-\text { Benzenediol }}$

$$
\text { 2-Methyl- }
$$$$
\text { 4-Hexyl- }
$$

Total Number

of

Observations

1
1

43

Total
Maximum Concentration, $\mathrm{mg} / \mathrm{m}^{3}$

0.0055 0.0055

0.3041
Maximum

Concentration ppov

MW

124

124

194

1
1

Tank with
Maximum
Concentration

C-107

C-105

41

Note

$\left({ }^{*}\right)$ The positional, geometrical or stereo isomer has not been defined. 
Table 8a. Aldehydes

Compound
ALIPHATIC ALDEHYDES
Ethanal
Propanal
2-Methyl-
2,2-Dimethyl-
Butanal
2-Methyl-
3-Methyl-
Pentanal
2-Methyl-
3-Methyl-
Hexanal
3-Methyl-
2-Ethyl-
Heptanal
Octanal
Nonanal
Decanal
Undecanal
Dodecanal

\section{Total Number of \\ Observations}

41
9
2
2
107
3
3
15
2
2
41
3
4
33
28
31
17
3
3

\begin{tabular}{|c|c|}
\hline $\begin{array}{c}\begin{array}{c}\text { Maximum } \\
\text { Concentration, } \\
\mathrm{mg} / \mathrm{m}^{3}\end{array} \\
\end{array}$ & MW \\
\hline 0.700 & 44 \\
\hline 0.560 & 58 \\
\hline 0.011 & 72 \\
\hline 0.030 & 86 \\
\hline 72.000 & 72 \\
\hline 0.080 & 86 \\
\hline 0.110 & 86 \\
\hline 0.920 & 86 \\
\hline 0.230 & 100 \\
\hline 0.190 & 100 \\
\hline 5.700 & 100 \\
\hline 0.730 & 114 \\
\hline 0.190 & 128 \\
\hline 0.600 & 114 \\
\hline 2.600 & 128 \\
\hline 6.600 & 142 \\
\hline 0.031 & 156 \\
\hline 0.004 & 170 \\
\hline 0.006 & 184 \\
\hline
\end{tabular}

0.015

0.080

0.072

0.052

$\left({ }^{*}\right)$

0.070

0.310

0.010

0.420

0.018

0.054

0.024

0.032

92.4

382

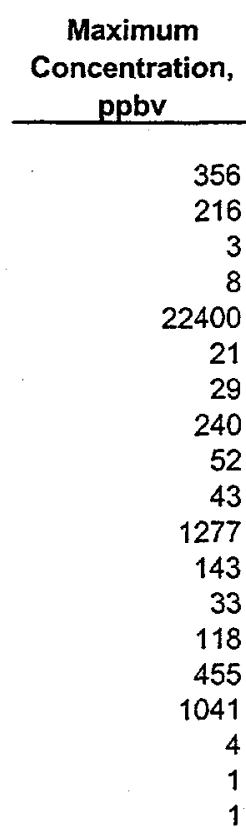

6

26

23

14

14

71
2

112

140

140

180

266

106

7

U-106

Total

26,681

Note

$\left.{ }^{\star}\right)$ The positional, geometrical or stereo isomer has not been defined. 
Table 8b. Aldehydes Observed in One Tank at Concentration Less Than $0.025 \mathrm{mg} / \mathrm{m}^{3}$

\begin{tabular}{|c|c|c|c|c|c|c|c|}
\hline Compound & & $\begin{array}{c}\text { Total Number } \\
\text { of } \\
\text { Observations }\end{array}$ & $\begin{array}{c}\text { Maximum } \\
\text { Concentration, } \\
\mathrm{mg} / \mathrm{m}^{3}\end{array}$ & MW & $\begin{array}{c}\text { Maximum } \\
\text { Concentration, } \\
\text { ppbv } \\
\end{array}$ & $\begin{array}{c}\text { Tank with } \\
\text { Maximum } \\
\text { Concentration }\end{array}$ & $\begin{array}{c}\text { TWINS2 } \\
\text { (CAS) } \\
\text { Number }\end{array}$ \\
\hline \multicolumn{8}{|l|}{ ALKANALS } \\
\hline \multicolumn{8}{|l|}{ Octanal } \\
\hline 7-Hydroxy-3,7-dimethyl- & & 1 & 0.001 & 156 & 0.1 & $T-107$ & $107-75-5$ \\
\hline Tridecanal & & 1 & 0.002 & 198 & 0.2 & C-107 & $10486-19-8$ \\
\hline Hexadecanal & & 1 & 0.0047 & 240 & 0.4 & BY-111 & $629-80-1$ \\
\hline ALKENALS & & & & & & & \\
\hline Z-3-Hexenal & & 1 & 0.021 & 98 & 5 & $U-103$ & $6789-80-6$ \\
\hline E-2-Octeneal & & 1 & 0.009 & 112 & 2 & $A X-101$ & $2548-87-0$ \\
\hline E,E-2,4-Nonadienal & & 1 & 0.0054 & 124 & 1 & $A X-101$ & $5910-87-2$ \\
\hline E-2-Deceneal & & 1 & 0.0043 & 154 & 1 & $A X-101$ & $3913-81-3$ \\
\hline 2-Dodeceneal & $(*)$ & 1 & 0.004 & 182 & 0.5 & C-109 & $4826-62-4$ \\
\hline \multicolumn{8}{|l|}{$\begin{array}{l}\text { CYCLIC COMPOUNDS } \\
\text { Cyclohexanal }\end{array}$} \\
\hline 4-(1-methylethyl)- & $(*)$ & 1 & 0.021 & 154 & 3 & $C-110$ & UAD010-02 \\
\hline Cyclohex-3-en-1-al & & & & & & & \\
\hline 1,3,4-Trimethyl- & $(*)$ & 1 & 0.005 & 152 & 1 & TY-104 & $40702-26-9$ \\
\hline \multicolumn{8}{|l|}{ BENZENE DERIVATIVES } \\
\hline \multicolumn{8}{|l|}{ Benzaldehyde } \\
\hline 3-Phenoxy- & & 1 & 0.002 & 182 & 0.2 & $\mathrm{C}-107$ & $39515-51-0$ \\
\hline Total & & 11 & 0.079 & & 14 & & \\
\hline
\end{tabular}

Note

(*) The positional, geometrical or stereo isomer has not been defined. 
Table 9a. Ketones (Page 1 of 2)

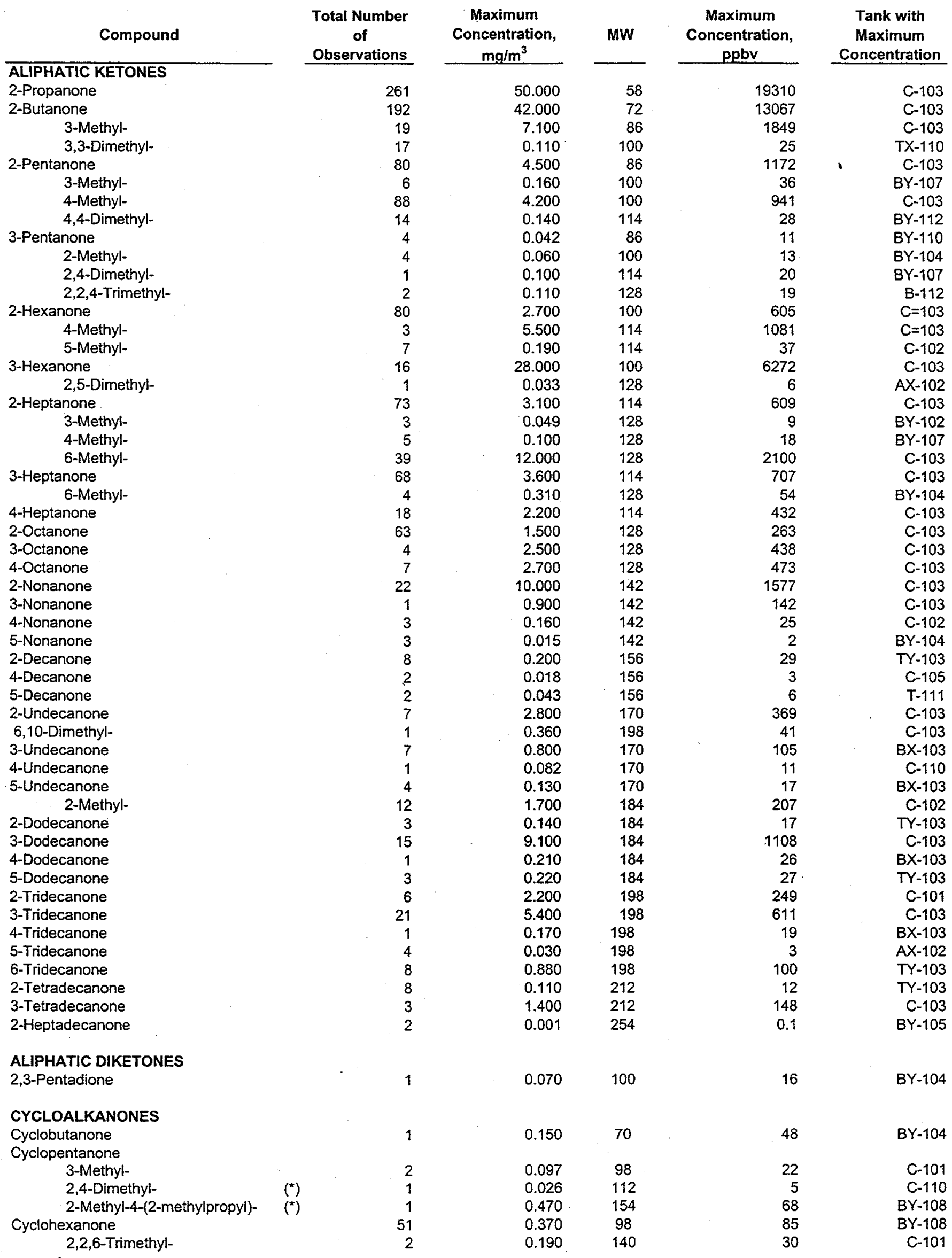


Table 9a. Ketones (Page 2 of 2)

\section{Compound}

3,3,5-Trimethyl-

5-Methyl-2-(1-methylethenyl)-

Bicyclo[4.1.0]heptan-3-one

4,7,7-Trimethyl-

\section{ALKENEONES}

3-Buten-2-one

3-Methyl-

3-Penten-2-one

$$
\text { 4-Methyl- }
$$

3-Hexen-2-one

3-Hepten-2-one 3-Methyl-

4-Methyl-

4-Hepten-3-one

$$
\text { 5-Ethyl-2,4-dimethyl- }
$$

1-Octen-3-one

4-Octen-3-one

2-Nonen-4-one

Z-5,9-Undecadien-2-one 6,10-Dimethyl-

2-Pentanone

$$
\text { 4-Cyclohexylidene-3,3-diethyl- }
$$

2-Hexanone

3-Cyclohexylidene-4-ethyl-
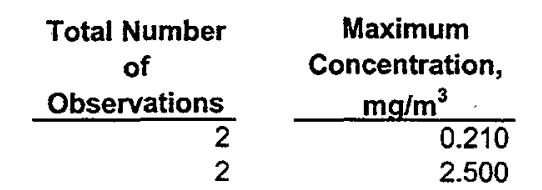

\begin{tabular}{r} 
MW \\
\hline 140 \\
152
\end{tabular}

$\begin{gathered}\text { Maximum } \\ \text { Concentration, } \\ \text { ppbv }\end{gathered}$
368

Tank with

Maximum

Concentration

1152

(*)

(*)

3

(*)

$\left({ }^{*}\right)$

1

1

$0.140 \quad 126$

$0.040 \quad 126$

$0.230 \quad 168$

$0.034 \quad 126$

$0.020 \quad 126$

$\left({ }^{*}\right)$

(*)

$0.063 \quad 140$

0.007

194

0.170

222

0.270

208

2.400

120

0.280

134

0.017

180

0.026

220

1363

222.69

57,142 368

85

21

20

112

25

C-101

C-204

C-204

C-103

BY-104

AX-102

AX-102

AX-102

TX-118

BY -107

BY-106

BY-104

U-106

C-107

BY-108

C-102

29

448

C-103

47

C-101

C-110

SX-107

Total

Note

(*) The positional, geometrical or stereo isomer has not been defined. 
Table 9b. Ketones Observed in One Tank at Concentration Less Than $0.025 \mathrm{mg} / \mathrm{m}^{3}$ (Page 1 of 1 )

\begin{tabular}{|c|c|c|c|c|c|c|c|}
\hline Compound & & $\begin{array}{c}\text { Total Number } \\
\text { of } \\
\text { Observations }\end{array}$ & $\begin{array}{c}\text { Maximum } \\
\text { Concentration, } \\
\mathrm{mg}^{3} \mathrm{~m}^{3} \\
\end{array}$ & MW & $\begin{array}{c}\text { Maximum } \\
\text { Concentration, } \\
\text { ppbr }\end{array}$ & $\begin{array}{c}\text { Tank with } \\
\text { Maximum } \\
\text { Concentration }\end{array}$ & $\begin{array}{l}\text { TWins2 } \\
\text { (CAS) } \\
\text { Number }\end{array}$ \\
\hline ALIPHATIC KETONES & & & & & & & \\
\hline $\begin{array}{l}\text { Pentanone } \\
\text { 4-Hydroxy-4-methyl- } \\
\text { 2-Hexanone }\end{array}$ & $(*)$ & 1 & 0.0180 & 116 & 3 & $T X-118$ & UKE006-03 \\
\hline 6-Methoxy- & & 1 & 0.0007 & 130 & 0.1 & $T \gamma-104$ & $29006-00-6$ \\
\hline 3-Hexanone & & & & & & & \\
\hline 4-Ethyl- & & 1 & 0.0034 & 128 & 1 & $A X-102$ & \\
\hline 2-Heptanone & & 1 & 0.0088 & 114 & 2 & $A X-102$ & $623-56-3$ \\
\hline $\begin{array}{l}\text { 2-Heptanone } \\
\text { 4,6-Dimethyi- } \\
\text { 4-Heptanone }\end{array}$ & & 1 & 0.0061 & 142 & 1 & BY -112 & $19549-80-5$ \\
\hline 4-Octanone & & 1 & 0.0200 & 128 & 4 & 8-103 & $15726-15-5$ \\
\hline $\begin{array}{l}\text { 3-Methyl- } \\
\text { 2-Decanone }\end{array}$ & & $\hat{\imath}$ & 0.0180 & 142 & 3 & $A X-102$ & $20754-04-5$ \\
\hline $\begin{array}{l}\text { 5,9-Dimethyl- } \\
\text { 4-Dodecanone }\end{array}$ & & 1 & 0.0240 & 184 & 3 & C-104 & $33933-82-3$ \\
\hline $\begin{array}{l}\text { 11-Methyl- } \\
\text { 2-Pentadecanone }\end{array}$ & & 1 & 0.0250 & 198 & 3 & $T Y-104$ & $29366-35-6$ \\
\hline 6,10,14-Trimethy|- & & 1 & 0.0057 & 268 & 0.5 & BT-105 & $502-69-2$ \\
\hline 4-Tetradecanone & & 1 & 0.0130 & 212 & 1 & $T \gamma-104$ & $26496-20-8$ \\
\hline $\begin{array}{l}\text { CYCLOALKANONES } \\
\text { Cyclobutanone }\end{array}$ & & & & & & & \\
\hline 2-Ethyl- & & 1 & 0.0220 & 98 & 5 & C-110 & $10374-14-8$ \\
\hline 3,3-Dimethyl- & & 1 & 0.0047 & 98 & 1 & $T X-118$ & $1192-33-2$ \\
\hline 2,3,3-Trimethyl- & $(*)$ & 1 & 0.0110 & 112 & 2 & $B Y-106$ & $28290-01-9$ \\
\hline Cyclopentanone & & & & & & & \\
\hline trans-3,4-Dimethy!- & & 1 & 0.0006 & 112 & 0.1 & $T Y-104$ & $19550-73-3$ \\
\hline 2,2,5-Trimethyl- & & 1 & 0.0150 & 126 & 3 & B-103 & $4573-09-5$ \\
\hline Cyclohexanone & & & & & & & \\
\hline 3-Methyl- & & 1 & 0.0210 & 112 & 4 & $T-111$ & $591-24-2$ \\
\hline 2,6-Dimethyl- & (") & 1 & 0.0040 & 126 & $i$ & BY-106 & $16519-68-9$ \\
\hline 4-Hydroxy-4-methyl- & & 1 & 0.0020 & 128 & 0.4 & C-107 & $17429-02-6$ \\
\hline ALIPHATIC DIONES & & & & & & & \\
\hline 2,5-Hexandione & & 1 & 0.0077 & 114 & 2 & $A x-102$ & $110-13-4$ \\
\hline $\begin{array}{l}\text { ACYCLIC AND CYCLIC ALKENEONES AN } \\
\text { 3-Hexen-2-one }\end{array}$ & No! & RELATED COMPC & & & & & \\
\hline $\begin{array}{l}\text { 5-Methyi- } \\
\text { 5-Hepten-2-one }\end{array}$ & $(*)$ & 1 & 0.0170 & 112 & 3 & $A \times-102$ & $5166-53-0$ \\
\hline 6-Methyl- & $(")$ & 1 & 0.0070 & 126 & 1 & $C-107$ & \\
\hline 7-Octen-2-one & & 1 & 0.0070 & 126 & 1 & $B \gamma-106$ & 35194-30-? \\
\hline 2-Methyl- & & 1 & 0.0060 & 154 & 1 & C-107 & $51756-19-5$ \\
\hline 10-Undecen-4-one & & & & & & & \\
\hline $\begin{array}{l}\text { 2,2,6,6-Tetramethyl- } \\
\text { 3-Cyclopenten-1-one. }\end{array}$ & (") & 1 & 0.0140 & 224 & 1 & A-102 & $42565-49-1$ \\
\hline $\begin{array}{l}\text { 2,3,4-Trimethyl- } \\
\text { Cyclohexanone }\end{array}$ & & 1 & 0.0045 & 124. & 1 & $T X-118$ & $83321-16-8$ \\
\hline $\begin{array}{l}\text { 2,5-Dimethyl-2-(1-methylethenyl)- } \\
\text { 2-Cyclohexen-1-one }\end{array}$ & & 1 & 0.0030 & 166 & 0.4 & C-107 & $6711-26-8$ \\
\hline 4,5-Dimethy|- & $(*)$ & 1 & 0.0250 & 124 & 5 & BY-102 & $5715-25-3$ \\
\hline $\begin{array}{l}\text { 4-Ethyl-3,4-dimethyl- } \\
\text { 3,4-Undecadien-2,10-dione }\end{array}$ & (") & 1 & 0.0033 & 152 & 0 & BY-106 & $17622-46-7$ \\
\hline $\begin{array}{c}\text { 6,6-Dimethyl- } \\
\text { E-5,9-Undecadien-2-one }\end{array}$ & & 1 & 0.0020 & 208 & 0.2 & $B Y=106$ & $52588-78-0 \mathrm{M}$ \\
\hline 6,10-Dimethyl- & & 1 & 0.0007 & 194 & 0.1 & $A X-103$ & $3796-70-1$ \\
\hline $\begin{array}{l}\text { AROMATIC KETONES AND QUINONES } \\
\text { 1H-Inden-1-one }\end{array}$ & & & & & . & & \\
\hline Ethanone 2,3-Dihydro-3,3-dimethyl- & & 1 & 0.0030 & 160 & 0.4 & C-107 & $26465-81-6$ \\
\hline 1-(2,4,5-Trimethylphenyl)- & & 1 & 0.0170 & 162 & 2 & $T-111$ & \\
\hline $\begin{array}{l}\text { 1-(3-Methylphenyl)- } \\
\text { 1-(5,6,7,8-Tetrahydro-3,5,5,6,8,8- }\end{array}$ & & 1 & 0.0020 & 134 & 0.3 & $T-107$ & $585-74-0$ \\
\hline hexamethyl-2-naphthenyl)- & & 1 & 0.0010 & 258 & 0.1 & C-109 & $1506-02-1$ \\
\hline bis- $1,1^{\prime}-(1,4-$-phenylene $)$ & & 1 & 0.0030 & 162 & 0.4 & TY-104 & $1009-61-6$ \\
\hline 1.4-Cyclohexadienone & & 1 & 0.0170 & 108 & 4 & $U-106$ & $637-88-7$ \\
\hline
\end{tabular}

Note

(*) The positional, geometrical or stereo isomer has not been defined. 
Table 10a. Acids

\begin{tabular}{|c|c|c|c|c|c|c|}
\hline Compound & & $\begin{array}{c}\text { Total Number } \\
\text { of } \\
\text { Observations }\end{array}$ & $\begin{array}{c}\text { Maximum } \\
\text { Concentration, } \\
\mathrm{mg} / \mathrm{m}^{3}\end{array}$ & MW & $\begin{array}{c}\text { Maximum } \\
\text { Concentration, } \\
\text { ppbv }\end{array}$ & $\begin{array}{c}\text { Tank with } \\
\text { Maximum } \\
\text { Concentration }\end{array}$ \\
\hline \multicolumn{7}{|l|}{ ALKANOIC ACIDS } \\
\hline Ethanoic & & 19 & 0.700 & 60 & 261 & C-104 \\
\hline Propanoic & & 4 & 0.017 & 74 & 5 & C-108 \\
\hline Butanoic & & 2 & 3.300 & 88 & 840 & C-103 \\
\hline Decanoic & & 2 & 0.003 & 172 & 0.4 & $c-111$ \\
\hline Dodecanoic & & 6 & 0.310 & 200 & 35 & BY-108 \\
\hline Tetradecanoic & & 22 & 4.000 & 228 & 393 & C-103 \\
\hline 12-Methyl- & & 2 & 0.010 & 242 & 1 & C-107 \\
\hline Pentadecanoic & & 13 & 2.400 & 242 & 222 & C-103 \\
\hline Hexadecanoic & & 30 & 4.200 & 256 & 368 & C-103 \\
\hline \multicolumn{7}{|l|}{ ALKENOIC ACIDS } \\
\hline Propenoic & & 1 & 4.400 & 72 & 1369 & $C-103$ \\
\hline Pentadec-14-enoic & $\left(^{*}\right)$ & 3 & 0.042 & 240 & 4 & BY -107 \\
\hline Hexadec-9-enoic & $(*)$ & 14 & 3.700 & 254 & 326 & $C-103$ \\
\hline Z-Octadec-9-enoic & & 5 & 0.070 & 282 & 6 & BY -107 \\
\hline Total & & 123 & 23.15 & & 3830 & ‘ \\
\hline
\end{tabular}

Note

$\left(^{*}\right)$ The positional, geometrical or stereo isomer has not been defined. 
Table 10b. Acids Observed in One Tank at Concentration Less Than $0.025 \mathrm{mg} / \mathrm{m}^{3}$

\begin{tabular}{|c|c|c|c|c|c|c|}
\hline Compound & $\begin{array}{c}\text { Total Number } \\
\text { of } \\
\text { Observations }\end{array}$ & $\begin{array}{c}\text { Maximum } \\
\text { Concentration, } \\
\mathrm{mg} / \mathrm{m}^{3}\end{array}$ & MW & $\begin{array}{c}\text { Maximum } \\
\text { Concentration, } \\
\text { ppbv }\end{array}$ & $\begin{array}{c}\text { Tank with } \\
\text { Maximum } \\
\text { Concentration }\end{array}$ & $\begin{array}{l}\text { TWINS2 } \\
\text { (CAS) } \\
\text { Number }\end{array}$ \\
\hline Hexanoic Acid & 1 & 0.0040 & $\overline{116}$ & 1 & C-109 & $142-62-1$ \\
\hline 2-Ethyl- & 1 & 0.0020 & 144 & 0.3 & C-109 & $149-57-5$ \\
\hline $\begin{array}{l}\text { Octadecanoic Acid } \\
\text { Cyclohexanecarboxylic Acid }\end{array}$ & 1 & 0.0040 & 284 & 0.3 & C-108 & $57-11-4$ \\
\hline trans-2-(1,1-Dimethylethyi)- & 1 & 0.0020 & 170 & 0.3 & C-112 & $27392-16-1$ \\
\hline Total & 4 & 0.0120 & & 2 & & \\
\hline
\end{tabular}


Table 11a. Esters

Compound

ALKANE ESTERS

Methanoic Acid Ester

2-Methylpropyl

Butyl

2,6-Dimethylhept-5-en-2-ol

$\left({ }^{\star}\right)$

Ethanoic Acid Ester

Methyl

Ethyl

2-Propenyl

Butyl

Hexadecanyl

Propanoic Acid Ester

Butyl

Octyl

2-Methylpropanoic Acid Ester

Butyl

Butanoic Acid Ester

Butyl

E-2-Hexenyl

Butanedioic Acid Ester

Diethyl

Hexandioic Acid Ester

Dioctyl

2-Ethylhexyl-

E-Hexa-2-enoic Acid Ester

Heptanoic Acid Ester Butyl

Tetradecanoic Acid Ester

1-Methylethyl

Butyl

Hexadecanoic Acid Ester 1-Methylethyl

Octadecanoic Acid Ester Butyl

Total Number

$$
\text { of }
$$

Observations
2-Propenyl

bis(2-Ethylhexyl)

E-2-Hexenyl

Maximum

Concentration, $\mathrm{mg} / \mathrm{m}^{3}$

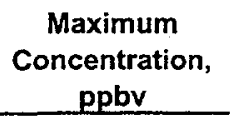

$$
0.300
$$

4.100

3.300

0.370

0.140

47.000

4.600

17.000

0.054

1.100

0.030

0.026

2.600

0.040

5.200

1.600

0.030

0.064

0.031

0.009

2.000

2.500

0.440

0.029

1.300

0.006

0.080

222

278

304

Butyl Cyclohexyl

2

C-103

BY-108

U-103

C-103

C-103

C-103

BY -107

C-103

U-106

AX-102

C-103

BY-105

C-103

C-103

B-103

S-105

C-102

C-105

C-103

C-103

BY-108

C-105

U-106

C-104

S-110

PHOSPHATE ESTERS

Butylphosphoric Acid Ester

$$
\text { Dibutyl }
$$

0.780

250

70

C-103

Phosphoric Acid Ester Tributyl

6.400

266

539

C-103

Note

(*) The positional, geometrical or stereo isomer has not been defined. 
Table 11b. Esters Observed in One Tank at Concentration Less Than $0.025 \mathrm{mg} / \mathrm{m}^{3}$

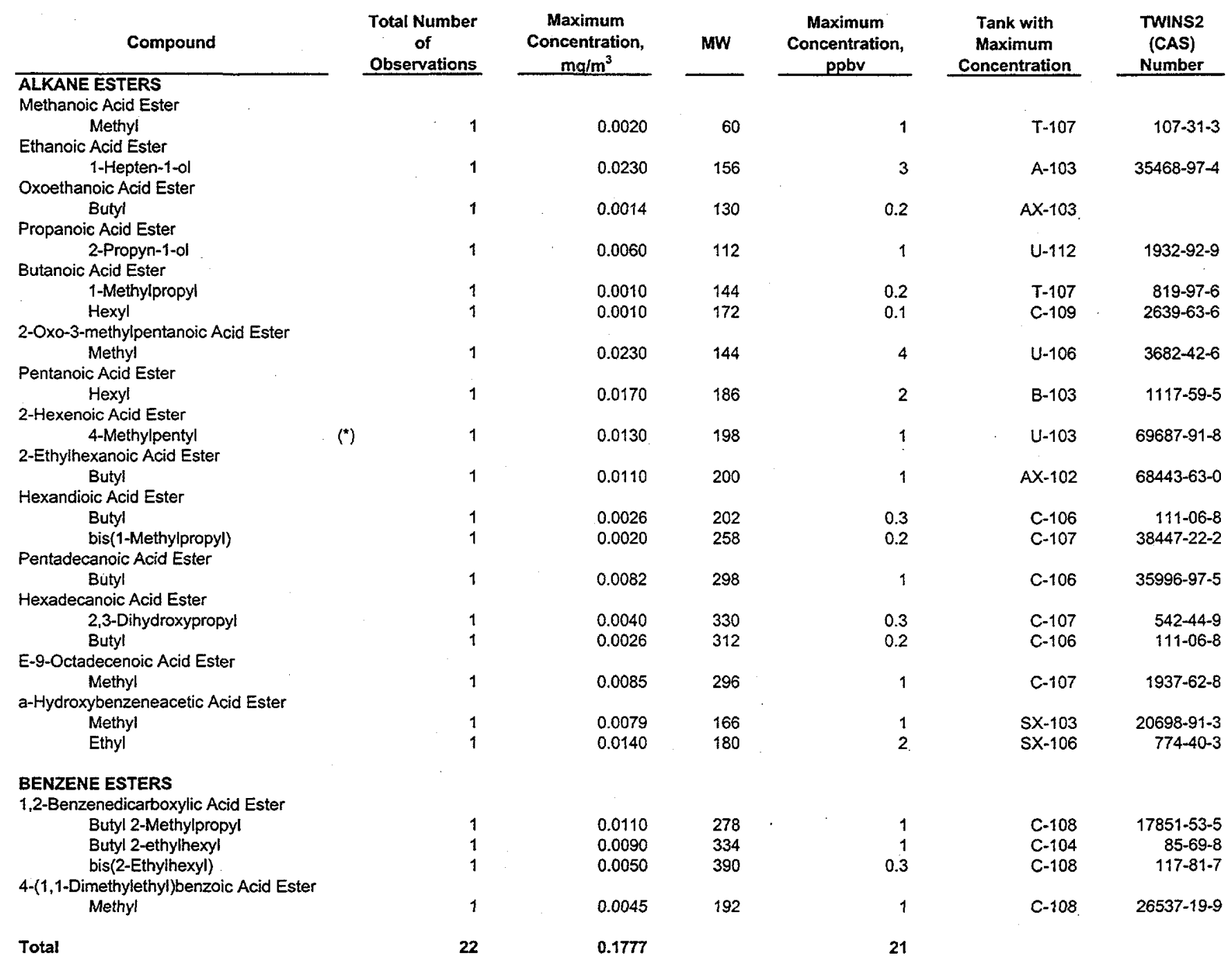

Note

(*) The positional, geometrical or stereo isomer has not been defined. 
Table 12a. Nitriles

\begin{tabular}{|c|c|c|c|c|c|c|}
\hline Compound & & $\begin{array}{c}\begin{array}{c}\text { Total Number } \\
\text { of }\end{array} \\
\text { Observations } \\
\end{array}$ & $\begin{array}{c}\text { Maximum } \\
\text { Concentration, } \\
\mathrm{mg} / \mathrm{m}^{3} \\
\end{array}$ & MW & $\begin{array}{c}\text { Maximum } \\
\text { Concentration, } \\
\text { ppbv } \\
\end{array}$ & $\begin{array}{c}\text { Tank with } \\
\text { Maximum } \\
\text { Concentration } \\
\end{array}$ \\
\hline \multicolumn{7}{|l|}{ ALKANE NITRILES } \\
\hline Acetonitrile & & 205 & 23.400 & 41 & 12784 & C-103 \\
\hline Propanenitrile & & 163 & 13.000 & 55 & 5295 & $C-103$ \\
\hline 2-Methyl- & & 2 & 0.060 & 69 & 19 & BY -104 \\
\hline 2,2-Dimethyl- & & 3 & 0.077 & 83 & 21 & $T X-111$ \\
\hline Butanenitrile & & 127 & 7.900 & 69 & 2565 & C-103 \\
\hline Pentanenitrile & & 114 & 4.000 & 83 & 1080 & $C-103$ \\
\hline Hexanenitrile & & 117 & 3.700 & 97 & 854 & C-103 \\
\hline Heptanenitrile & & 51 & 3.200 & 111 & 646 & C-103 \\
\hline Octanenitrile & & 23 & 1.900 & 125 & 340 & $C-103$ \\
\hline Nonanenitrile & & 19 & 0.920 & 139 & 148 & C-103 \\
\hline Decanenitrile & & 2 & 0.011 & 153 & 2 & $U-106$ \\
\hline Undecanenitrile & & 2 & 0.002 & 167 & 0.3 & TY-104 \\
\hline Tridecanenitrile & & 1 & 0.089 & 181 & 11 & BY -107 \\
\hline \multicolumn{7}{|l|}{ ALKENE NITRILES } \\
\hline 2-Propenenitrile & & 3 & 0.027 & 53 & 11 & $T X-118$ \\
\hline 2-Methyl- & & 1 & 0.110 & 67 & 37 & C-101 \\
\hline 2-Butenenitrile & $\left({ }^{*}\right)$ & 3 & 0.017 & 55 & 7 & $C-110$ \\
\hline 3-Butenenitrile & $(*)$ & 2 & 0.062 & 55 & 25 & $B Y-106$ \\
\hline \multicolumn{7}{|c|}{ CYCLOALKANE NITRILES } \\
\hline Cyclopropanenitrile & & 2 & 0.022 & 53 & 9 & C-107 \\
\hline \multicolumn{7}{|l|}{ AROMATIC NITRILES } \\
\hline Benzonitrile & & 5 & 0.075 & 103 & 16 & $C-110$ \\
\hline Total & & 845 & 58.57 & & 23,871 & \\
\hline
\end{tabular}

Notes

(*) The positional, geometrical or stereo isomer has not been defined. 
Table 12b. Nitriles Observed in One Tank at Concentration Less Than $0.025 \mathrm{mg} / \mathrm{m}^{3}$

\begin{tabular}{|c|c|c|c|c|c|c|c|}
\hline Compound & & $\begin{array}{c}\text { Total Number } \\
\text { of } \\
\text { Observations } \\
\end{array}$ & $\begin{array}{c}\text { Maximum } \\
\text { Concentration, } \\
\mathrm{mg} / \mathrm{m}^{3} \\
\end{array}$ & MW & $\begin{array}{c}\text { Maximum } \\
\text { Concentration, } \\
\text { ppbv } \\
\end{array}$ & $\begin{array}{c}\text { Tank with } \\
\text { Maximum } \\
\text { Concentration } \\
\end{array}$ & $\begin{array}{l}\text { TWINS2 } \\
\text { (CAS) } \\
\text { Number }\end{array}$ \\
\hline \multicolumn{8}{|l|}{ Acetonitrile } \\
\hline Hydroxy- & & 1 & 0.0029 & 57 & 1 & TY-104 & $107-16-4$ \\
\hline Undecanenitrile & & 2 & 0.0022 & 167 & 0.3 & TY-104 & $2244-07-7$ \\
\hline 2,4-Pentadienenitrile & $\left({ }^{\star}\right)$ & 1 & 0.0030 & 79 & 1 & $\mathrm{U}-112$ & $1615-70-9$ \\
\hline Total & & 4 & 0.0081 & & 2 & & \\
\hline
\end{tabular}

Note

$\left(^{*}\right)$ The positional, geometrical or stereo isomer has not been defined. 
Table 13a. Amines and Amides

\section{Compound}

ALIPHATIC AMINES

Methanamine

\section{N, N-Dimethyl-}

$\mathrm{N}$-(1-Methylbutylidene)-

Butanamine

$$
\mathrm{N} \text {-Ethylidene- }
$$

1-Pentanamine

5-Hydroxy-
(*)

of

Observations

(*)

2
Total Number

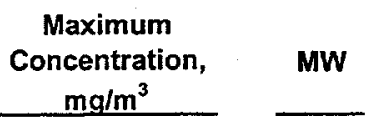

1.700

0.580

0.035

0.003

0.140

0.180

2.100

0.043

0.032

$$
\text { 1-Methyl- }
$$

1,3,5,7-Tetraazatri(3.3.1.1(3,7))decane

\section{AROMATIC AMINES}

Benzeneamine

$$
\text { N-Phenyl }
$$

10

0.230

169

\section{ALIPHATIC AMIDES}

Cyanamide

Formamide

$$
\text { Dimethyl- }
$$

N-(2-Methylpropyl)-

Acetamide

$$
\text { N-Butyl- }
$$

Butanamide

N,N-Dimethyl-

Octanamide $\mathrm{N}$-(2-Hydroxyethyl)-

CYCLIC ALIPHATIC AMIDES

2-Pyrrolidinone 1-Methyl-

2,5-Pyrrolidenedione 1-Methyl-

Total
1

3

1

2

2

2

3

2

5

1

2

64
0.130

0.008

0.026

0.060

0.084

0.050

0.077

0.022

0.960

0.110

0.028

6.60
30

BY-108

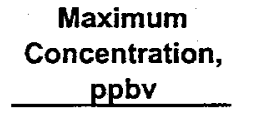

645

131

13

U-106

TY-101

S-102

A-101

C-103

U-106

TY-101

5

57

663

U-106

BY-112

BY-106

A-101

AX-102

C-105

AX-102

A-103

253

C- 103

BY-109

C-108

Note

$\left.{ }^{\star}\right)$ The positional, geometrical or stereo isomer has not been defined. 
Table 13b. Amines and Amides Observed in One Tank at a Concentration Less Than $0.025 \mathrm{mg} / \mathrm{m}^{3}$

\section{Compound}

ACYCLIC ALIPHATIC AMINES

Methanamine

$N-(\{-$ Methylhexylididene)-

Propanamine

$\mathrm{N}-(4-\mathrm{H} y d r o x y-2-m e t h o x y p h e n y l)$ -

3-Cyano-

1-Butanone

4-(Dimethylamino)-1-phenyl-

1-Octadecanamine $\mathrm{N}, \mathrm{N}$-Dimethyl-

1,4-Butanediamine

ACYCLIC ALIPHATIC AMIDES

Acetamide

Butanamide

$$
\text { N-Methyl- }
$$

N-Hexyl-

Nonanamide

Decanamide

$$
\mathrm{N} \text {-(2-Hydroxyethyl) }
$$

Dodecanamide N-(2-Hydroxyethyl)

Hexadecanamide

Total Number

of

Observations
N-Methyl-

Total
CYCLIC AMIDES

3-Piperidinecarboxamide
Concentration. $\mathrm{mg} / \mathrm{m}^{3}$
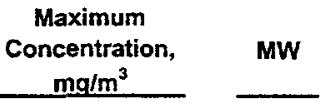
Maximum
Concentration ppbv

0.0130

0.0210

127
167

0.0023

84

0.0160

215

0.0040

0.0012

297

88

0.0012

73

0.0004

0.0080

171

157

0.0055

215

0.0078

0.0030

243

255

0.0083

121

0.0020

135

0.0094

142

0.1031

Tank with

Maximum

Concentration

0.3

0.4

0.1

1

1

1

0.3

2

0

15

14
C-104

$\mathrm{T}-107$

T-107

C-110

TY-104

TY-104

A-102

C-107

578-54-1

(CAS)

Number

22058-71-5

1196-92-5

$151-18-8$

$3760-63-2$

124-28-7

$110-60-1$

$79-16-3$

0264-17-2 1120-07-6

142-78-9

$629-54-9$

$T Y-104$

C-108

613-93-4

SX-106

5115-98-0 
Table 14a. Nitrous and Nitric Acid Esters, Nitrous and Nitro Compounds

\begin{tabular}{|c|c|c|c|c|c|}
\hline Compound & $\begin{array}{c}\text { Total Number } \\
\text { of } \\
\text { Observations }\end{array}$ & $\begin{array}{c}\text { Maximum } \\
\text { Concentration, } \\
\mathrm{mg} / \mathrm{m}^{3}\end{array}$ & MW & $\begin{array}{c}\text { Maximum } \\
\text { Concentration, } \\
\text { ppbv }\end{array}$ & $\begin{array}{c}\text { Tank with } \\
\text { Maximum } \\
\text { Concentration }\end{array}$ \\
\hline \multicolumn{6}{|l|}{$\begin{array}{l}\text { NITROUS ACID ESTERS } \\
\text { AlCO }\end{array}$} \\
\hline Methyl & 11 & 0.870 & 61 & 319 & B-103 \\
\hline Butyl & 6 & 0.760 & 103 & 165 & A-101 \\
\hline \multicolumn{6}{|l|}{ NITRIC ACID ESTERS } \\
\hline \multicolumn{6}{|l|}{ Alcohol Component } \\
\hline Methyl & 14 & 0.200 & 77 & 58 & $C-112$ \\
\hline Ethyl & 23 & 0.450 & 91 & 111 & $B X-103$ \\
\hline 1-Methylethyl & 4 & 0.150 & 105 & 32 & C-102 \\
\hline Propyl & 28 & 1.100 & 105 & 235 & TY-105 \\
\hline 2,2-Dimethyl- & 6 & 0.170 & 133 & 29 & $A X-102$ \\
\hline Butyl & 27 & 0.810 & 119 & 152 & TY-105 \\
\hline 3-Methyl- & 5 & 0.250 & 133 & 42 & $B X-103$ \\
\hline Pentyl & 12 & 0.310 & 133 & 52 & B-103 \\
\hline Hexyl & 12 & 0.620 & 147 & 94 & BX-103 \\
\hline Heptyl & 3 & 0.039 & 161 & 5 & $T X-111$ \\
\hline \multicolumn{6}{|l|}{ Diol and Triol Component } \\
\hline 1,3-Propanediol & 1 & 13.000 & 178 & 1636 & C-204 \\
\hline 1,4-Butanediol & 4 & 2.100 & 192 & 245 & $C-103$ \\
\hline 1,5-Pentanediol & 4 & 0.028 & 206 & 3 & B-103 \\
\hline \multicolumn{6}{|l|}{ Propane-1,2,3-triol } \\
\hline 1-Nitrate & 1 & 0.160 & 149 & 24 & C-204 \\
\hline 1,3-Dinitrate & 2 & 0.077 & 192 & 9 & $T X-106$ \\
\hline \multicolumn{6}{|l|}{ NITRO COMPOUNDS } \\
\hline \multicolumn{6}{|l|}{ Methane } \\
\hline Nitro- & 4 & 0.140 & 61 & 51 & U-112 \\
\hline Trinitrofluoro- & 2 & 0.110 & 169 & 15 & $\mathrm{BX}-103$ \\
\hline \multicolumn{6}{|l|}{ Propane } \\
\hline 1-Nitro- & 2 & 0.130 & 89 & 33 & TY-105 \\
\hline \multirow{2}{*}{\multicolumn{6}{|c|}{ Butane }} \\
\hline & & & & & \\
\hline 1-Nitro- & 6 & 1.800 & 103 & 391 & $C-204$ \\
\hline \multicolumn{6}{|l|}{ Benzene } \\
\hline 1-Nitro & 2 & 0.015 & 123 & 3 & $T X-118$ \\
\hline 1-Nitro-4-methyl- & 2 & 0.002 & 137 & 0.3 & $\mathrm{~T}-107$ \\
\hline \multicolumn{6}{|l|}{ NITROSO COMPOUNDS } \\
\hline Nitrosomethane & 1 & 0.190 & 45 & 95 & $T X-118$ \\
\hline 4-Nitrosomorpholine & 1 & 0.050 & 100 & 11 & $U-108$ \\
\hline \multicolumn{6}{|l|}{ Methanamine } \\
\hline $\mathrm{N}$-Methyl-N-nitroso- & 26 & 0.270 & 74 & 82 & U-108 \\
\hline Total & 235 & 23.961 & & 3928 & \\
\hline
\end{tabular}


Table 14b. Nitrous and Nitric Acid Esters, Nitrous and Nitro Compounds Observed in One Tank at a Concentration Less Than $0.025 \mathrm{mg} / \mathrm{m}^{3}$

\begin{tabular}{|c|c|c|c|c|c|c|}
\hline Compound & $\begin{array}{c}\begin{array}{c}\text { Total Number } \\
\text { of }\end{array} \\
\text { Observations } \\
\end{array}$ & $\begin{array}{c}\text { Maximum } \\
\text { Concentration, } \\
\mathrm{mg} / \mathrm{m}^{3} \\
\end{array}$ & MW & $\begin{array}{c}\text { Maximum } \\
\text { Concentration, } \\
\text { ppbv } \\
\end{array}$ & $\begin{array}{c}\text { Tank with } \\
\text { Maximum } \\
\text { Concentration }\end{array}$ & $\begin{array}{l}\text { TWINS2 } \\
\text { (CAS) } \\
\text { Number }\end{array}$ \\
\hline \multicolumn{7}{|l|}{$\begin{array}{l}\text { NITRIC ACID ESTERS } \\
\text { Nitrate }\end{array}$} \\
\hline 2-Methylpropyl & 1 & 0.0022 & 119 & 0.4 & $T X-105$ & $543-29-3$ \\
\hline Nonyl & 1 & 0.0014 & 189 & 0.2 & $T Y-104$ & 20633-13-0 \\
\hline Decyl & 1 & 0.0086 & 203 & 1 & TX-118 & $2050-78-4$ \\
\hline \multirow{2}{*}{\multicolumn{7}{|c|}{$\begin{array}{l}\text { NITRO COMPOUNDS } \\
\text { Propane }\end{array}$}} \\
\hline & & & & & & \\
\hline Butane ${ }^{2-M e t h y l-1-n i t r o}$ & 1 & 0.0090 & 103 & 2 & U-112 & $625-74-1$ \\
\hline 2-Nitro- & 1 & 0.0055 & 103 & 1 & $U-112$ & $600-24-8$ \\
\hline Z-1-Nitropropene & 1 & 0.0080 & 87 & 2 & $T Y-104$ & $27675-36-1$ \\
\hline \multicolumn{7}{|l|}{$\begin{array}{l}\text { BENZENE DERIVATIVES } \\
\text { Benzene }\end{array}$} \\
\hline 1-Nitro-4-chloro- & 1 & 0.0053 & 134 & 1 & C-107 & $100-00-5$ \\
\hline Total & 7 & 0.0400 & & 8 & & \\
\hline
\end{tabular}


Table 15a. Heterocycles (Page 1 of 1)

\begin{tabular}{|c|c|c|c|c|c|c|}
\hline Compound & & $\begin{array}{c}\text { Total Number } \\
\text { of } \\
\text { Observations }\end{array}$ & $\begin{array}{c}\text { Maximum } \\
\text { Concentration, } \\
\mathrm{mg} / \mathrm{m}^{3}\end{array}$ & MW & $\begin{array}{c}\text { Maximum } \\
\text { Concentration, } \\
\text { ppbv }\end{array}$ & $\begin{array}{c}\text { Tank with } \\
\text { Maximum } \\
\text { Concentration }\end{array}$ \\
\hline Oxirane & & 1 & 0.052 & $\overline{44}$ & 26 & TY-104 \\
\hline Ethyl & & 1 & 0.270 & 72 & 84 & B-103 \\
\hline Ethenyl- & & 4 & 2.500 & 70 & 800 & $B Y-108$ \\
\hline cis-2-Ethyl-3-propy! & & 1 & 0.160 & 114 & 31 & $B X-104$ \\
\hline Furan & & 9 & 9.700 & 68 & 3195 & $C-103$ \\
\hline 2-Methy- & & 2 & 3.700 & 82 & 1011 & C. 103 \\
\hline 2-Propyl- & & 2 & 0.046 & 110 & 9 & $B Y-109$ \\
\hline 2-Pentyl- & & 2 & 0.016 & 138 & 3 & $A X-103$ \\
\hline 2-Heptyl- & & 1 & 0.330 & 166 & 45 & BY-108 \\
\hline 2,5-Dimethyl- & & 1 & 0.040 & 96 & 9 & BY-104 \\
\hline 2-Ethyl-5-methyl- & & 2 & 0.050 & 110 & 10 & $A \times-102$ \\
\hline 2,3-Dihydrofuran & & 5 & 0.050 & 70 & 16 & $C-110$ \\
\hline 2,5-Dihydrofuran & & 5 & 2.700 & 70 & 864 & C-103 \\
\hline Tetrahydrofuran & & 166 & 16.000 & 72 & 4978 & $c-103$ \\
\hline 2,5-Diethyl- & (*) & 1 & 0.110 & 128 & 19 & A-101 \\
\hline 2,5-Dipropyl- & (") & 2 & 0.007 & 156 & 1 & $T Y-104$ \\
\hline Dihydro-2(3H)-Furanone & & 6 & 3.500 & 84 & 933 & C-103 \\
\hline 5-Methyt- & & 4 & 0.044 & 98 & 10 & $A X-102$ \\
\hline 5-Ethyl- & & 9 & 0.089 & 112 & 18 & U-106 \\
\hline 5-Propyl- & & 7 & 0.011 & 126 & 2 & C-104 \\
\hline 5-Butyl- & & 4 & 0.006 & 140 & 1 & $T \gamma-104$ \\
\hline 5-Hexyl- & & 2 & 0.006 & 168 & 1 & C-104 \\
\hline 3,5-Dimethyl- & & 2 & 0.710 & 112 & 142 & C-102 \\
\hline Benzofuranone & & & & & & \\
\hline 3a,4,5,6-tetrahydro-3a,6,6-trimethyl- & & 6 & 0.730 & 188 & 87 & BY-108 \\
\hline Tetrahydrofuranmethanol & & 1 & 0.075 & 102 & 16 & C-102 \\
\hline Furanacetaldehyde & & & & & & \\
\hline alpha-propyl & & 1 & 0.200 & 152 & 29 & BY-107 \\
\hline $\begin{array}{l}\text { 1,4-Dioxane } \\
\text { 2,3-Dioxabicyclo[2.2.2]oct-5-ene }\end{array}$ & & 8 & 0.060 & 88 & 15 & S-102 \\
\hline 1-Methyl-4-(1-methylethyl)- & & 1 & $0.400^{\circ}$ & 188 & 48 & C-103 \\
\hline 2,2-Bioxepane & & 6 & 0.054 & 72 & 17 & $A x-102$ \\
\hline 2H-Pyran-2-one & & & & & & \\
\hline Tetrahydro-5,6-dimethyl- & & 5 & 1.200 & 128 & 210 & BY-108 \\
\hline 1H-Pyrrole & & 2 & 0.012 & 67 & 4 & BY-106 \\
\hline Pyridine & & 96 & 0.430 & 79 & 122 & BY-108 \\
\hline 2-Methyl- & & 1 & 0.061 & 93 & 15 & C- 104 \\
\hline 3-methyl- & & 5 & 0.150 & 93 & 36 & C-101 \\
\hline 4-Methyl- & & 5 & 0.037 & 93 & 9 & C-105 \\
\hline 2-Ethyt- & & 2 & 0.011 & 107 & 2 & $A X-102$ \\
\hline 2,4-Dimethyl- & & 3 & 0.500 & 107 & 105 & C-103 \\
\hline 2,5-Dimethyl- & & 1 & 0.089 & 107 & 19 & C-104 \\
\hline 5-Ethyl-2-methyl- & & 2 & 0.014 & 121 & 3 & C-104 \\
\hline 1,2,3,6-Tetrahydropyridine & & 2 & 0.110 & 83 & 30 & $U-106$ \\
\hline Pyrimidine & & 4 & 0.390 & 80 & 109 & $\mathrm{U}-102$ \\
\hline 4-Methyl- & & 1 & 0.038 & 94 & 9 & $T X-111$ \\
\hline Pyrazine & & 40 & 0.390 & 80 & 109 & U-102 \\
\hline Methyl- & & 9 & 0.023 & 94 & 5 & $T X-111$ \\
\hline Ethyt- & & 4 & 0.032 & 108 & 7 & $T X-111$ \\
\hline 2,3-Dimethyl- & & 3 & 0.015 & 108 & 3 & A-103 \\
\hline 1H-1,2,4-Triazole & & 2 & 0.027 & 69 & 9 & $U-103$ \\
\hline 1-Ethyl- & & 1 & 0.130 & 97 & 30 & BY-105 \\
\hline 1H-Pyrazole & & & & & & \\
\hline 3-Methyl- & & 3 & 0.230 & 82 & 63 & $B X-104$ \\
\hline 4,5-Dihydro-1H-pyrazole & & & & & & \\
\hline $\begin{array}{l}\text { 5-Methyl- } \\
\text { 4,5-Dihydrooxazole }\end{array}$ & & 1 & 0.067 & 96 & 16 & U-106 \\
\hline 2-Methyl- & & 8 & 0.062 & 99 & 14 & $\mathrm{U}-106$ \\
\hline 2-Propyl- & & 3 & 0.047 & 127 & 8 & S-102 \\
\hline 2-Oxazolidinone & & & & & & \\
\hline 5-Methyl-3-(2-propenyl)- & $(*)$ & 4 & 0.067 & 141 & 11 & U-106 \\
\hline Isothiazole & & 2 & 0.003 & 97 & 1 & C-112 \\
\hline Benzothiazole & & 4 & 0.060 & 135 & 10 & BY-106 \\
\hline 2-Phenyl- & & 4 & 0.062 & 211 & 7 & $s x-106$ \\
\hline Total & & 479 & 45.872 & & 13,385 & \\
\hline
\end{tabular}

Note

(") The positional, geometrical or stereo isomer has not been defined. 
Table 15b. Heterocycles Observed in One Tank at a Concentration Less Than $0.025 \mathrm{mg} / \mathrm{m}^{3}$

\begin{tabular}{|c|c|c|c|c|c|c|c|}
\hline Compound & & $\begin{array}{c}\text { Total Number } \\
\text { of } \\
\text { Observations } \\
\end{array}$ & $\begin{array}{c}\begin{array}{c}\text { Maximum } \\
\text { Concentration, } \\
\mathrm{ma} / \mathrm{m}^{3}\end{array} \\
\end{array}$ & MW & $\begin{array}{c}\text { Maximum } \\
\text { Concentration, } \\
\text { ppbv } \\
\end{array}$ & $\begin{array}{c}\text { Tank with } \\
\text { Maximum } \\
\text { Concentration } \\
\end{array}$ & $\begin{array}{c}\text { TWINS2 } \\
\text { (CAS) } \\
\text { Number } \\
\end{array}$ \\
\hline \multicolumn{8}{|l|}{$\overline{\text { Oxirane }}$} \\
\hline (1-Methylbutyl)- & & 1 & 0.0024 & 114 & 0.5 & S-111 & $53229-39-3$ \\
\hline 2-Methyl-2-pentyl- & & 1 & 0.0160 & 128 & 3 & $C-104$ & $53907-75-8$ \\
\hline \multicolumn{6}{|l|}{ Furan } & U-112 & $1779-19-7$ \\
\hline 2-Octyt & & 1 & 0.0065 & 180 & 1 & BY-106 & $4179-38-8$ \\
\hline 2-(2-Methyl-6-oxoheptyl)- & (") & 1 & 0.0045 & 194 & 1 & C-105 & $51595-87-0$ \\
\hline $\begin{array}{l}\text { 2-(3-Oxo-3-phenylprop-1-enyl)- } \\
\text { 2,3-Dihydrofuran }\end{array}$ & $(*)$ & 1 & 0.0051 & 198 & 1 & sX-106 & $717-21-5$ \\
\hline 3-(1,1-Dimethylethyl)- & & 1 & 0.0030 & 126 & 1 & BY-105 & $34314-82-4$ \\
\hline $\begin{array}{l}\text { 4-(1-Methylpropyl)- } \\
\text { Tetrahydrofuran }\end{array}$ & & 1 & 0.0055 & 126 & 1 & $A X-102$ & $34379-54-9$ \\
\hline trans-2,4-Dimethyl- & & 1 & 0.0140 & 100 & 3 & $B-103$ & $39168-02-0$ \\
\hline \multicolumn{8}{|l|}{ Dihydro-2(3H)-Furanone } \\
\hline 4,4-Dimethyl- & & 1 & 0.0060 & 114 & 1 & BY-112 & $13861-97-7$ \\
\hline 5-Ethenyl-5-methyl- & & 1 & 0.0025 & 126 & 0.4 & $5 X-106$ & $1073-11-6$ \\
\hline 5-Ethyl-5-methyl- & & 1 & 0.0079 & 128 & 1 & $T X-105$ & $2865-82-9$ \\
\hline 5-Pentyl- & & 1 & 0.0170 & 156 & 2 & $A X-102$ & $104-61-0$ \\
\hline $\begin{array}{l}\text { Dihydro-2,5-Furandione } \\
2,4(3 \mathrm{H}, 5 \mathrm{H}) \text {-Furandione }\end{array}$ & & 1 & 0.0089 & 100 & 2 & $5 X-103$ & $108-30-5$ \\
\hline $\begin{array}{c}\text { 3-Methyl- } \\
\text { Tetrahydrofuranmethanol }\end{array}$ & & 1 & 0.0180 & 114 & 4 & $A X-102$ & $1192-51-4$ \\
\hline trans-5-Methyl- & & 1 & 0.0034 & 116 & 1 & $T X-118$ & $54774-28-6$ \\
\hline Acetic acid ester & & 1 & 0.0030 & 144 & 0.5 & $T-107$ & $637-64-9$ \\
\hline \multicolumn{8}{|l|}{ 1,3-Dioxane } \\
\hline 4,4-Dimethyl- & & 1 & 0.0090 & 116 & 2 & C-105 & $766-15-4$ \\
\hline $\begin{array}{l}\text { 1,3-Benzodiox-2-one } \\
\text { 2H-Pyran }\end{array}$ & & 1 & 0.0023 & 150 & 0.3 & C-107 & $20192-66-9$ \\
\hline 3,4-Dihydro-2-carboxaldehyde & & 1 & 0.0079 & 112 & 2 & A-102 & $100-73-2$ \\
\hline Tetrahydro-2-(1,1-Dimethylethoxy)- & & 1 & 0.0050 & 158 & 1 & $T Y-104$ & $1927-69-1$ \\
\hline \multicolumn{8}{|l|}{ 2H-Pyran-2-one } \\
\hline Tetrahydro-6-hexyl- & & 1 & 0.0027 & 184 & 0.3 & S-111 & $710-04-3$ \\
\hline Tetrahydro-6,6-dimethyl- & & 1 & 0.0030 & 128 & 1 & $\mathrm{C}-112$ & $2610-95-9$ \\
\hline $\begin{array}{l}\text { Dihydro-2H-Pyran-3(4H)-ane } \\
\text { 1H-Pyrrole }\end{array}$ & & 1 & 0.0045 & 98 & 1. & $\mathrm{C}-107$ & $23462-75-1$ \\
\hline Pyridine 2,5-Dimethyl- & & 1 & 0.0110 & 95 & 3 & sX-106 & $625-84-3$ \\
\hline 3,4-Dimethyl- & & 1 & 0.0071 & 107 & 1 & S-111. & $583-58-4$ \\
\hline 2,6-Dimethyl- & & 1 & 0.0120 & 107 & 3 & $C-104$ & $108-48-5$ \\
\hline 2,3,6-Trimethyl- & & 1 & 0.0007 & 121 & 0.1 & $T Y-104$ & $1462-84-6$ \\
\hline \multicolumn{8}{|l|}{ Pyrazine } \\
\hline 2,5-Dimethyl- & & 1 & 0.0019 & 110 & 0.4 & S-111 & $123-32-0$ \\
\hline 2,5-Dimethyl-3-(3-methylbutyl)- & & 1 & 0.0100 & 178 & 1 & C-104 & $18433-98-2$ \\
\hline \multicolumn{8}{|l|}{ 4H-1,2,4-Triazole } \\
\hline $\begin{array}{l}\text { 3-Amino-4-ethyl- } \\
\text { 1H-Pyrazole }\end{array}$ & \multicolumn{6}{|c|}{ 1H-Pyrazole } & $42786-06-1$ \\
\hline $\begin{array}{c}\text { 1-Methyl- } \\
\text { 4,5-Dihydro- } 1 \text { H-pyrazole }\end{array}$ & & 1 & 0.0066 & 82 & 2 & TX-118 & $930-36-9$ \\
\hline 4,5-Dimethyl- & & 1 & 0.0240 & 98 & 5 & $U-106$ & 28019-94-5 \\
\hline 5-Propyl- & & 1 & 0.0230 & 112 & 5 & $U-103$ & $75011-90-4$ \\
\hline 1H-Pyrrolo(2,3-b)pyridine & & . & & & & & \\
\hline \multicolumn{8}{|l|}{ Hexahydro-1H-Azepin-1-amino } \\
\hline $\begin{array}{l}\text { N-Ethylidenehexa- } \\
\text { 1H-Imidazole }\end{array}$ & & 1 & 0.0083 & 140 & 1 & $\mathrm{C}-112$ & $75268-01-8$ \\
\hline 2-Methyl- & & 1 & 0.0016 & 82 & 0.4 & TY-104 & $693-98-1$ \\
\hline \multicolumn{8}{|l|}{$1 \mathrm{H}$-Indole } \\
\hline $\begin{array}{l}\text { 2-Phenyl- } \\
\text { 1-Pyrrolidinamine }\end{array}$ & & 1 & 0.0056 & 198 & 1 & $S X-106$ & $948-65-2$ \\
\hline 2-(Methoxymethyl)- & & 1 & 0.0018 & 130 & 0.3 & $T \gamma-104$ & $59983-39-0$ \\
\hline 1-Pyrrolidinecarboxylaldehyde & & 1 & 0.0006 & 99 & 0.1 & $T \gamma-104$ & $3760-54-1$ \\
\hline $4(3 H)$-Pyrimidinone & & 1 & 0.0220 & 96 & 5 & $B Y-102$ & $51953-17-4$ \\
\hline Totai & & 41 & 0.3281 & & 62 & & \\
\hline
\end{tabular}

(*) The positional, geometrical or stereo isomer has not been defined. 
Table 16a. Sulfur Compounds

\begin{tabular}{|c|c|c|c|c|c|}
\hline Compound & $\begin{array}{c}\text { Total Number } \\
\text { of } \\
\text { Observations } \\
\end{array}$ & $\begin{array}{c}\text { Maximum } \\
\text { Concentration, } \\
\mathrm{mg} / \mathrm{m}^{3} \\
\end{array}$ & MW & $\begin{array}{c}\text { Maximum } \\
\text { Concentration, } \\
\text { ppbv } \\
\end{array}$ & $\begin{array}{c}\text { Tank with } \\
\text { Maximum } \\
\text { Concentration }\end{array}$ \\
\hline \multicolumn{6}{|l|}{$\begin{array}{l}\text { THIOLS } \\
\text { 1-Propanethiol }\end{array}$} \\
\hline 2,2-Dimethyl- & 1 & 0.031 & 104 & 7 & C-105 \\
\hline \multicolumn{6}{|l|}{$\begin{array}{l}\text { SULFONES } \\
\text { Propane }\end{array}$} \\
\hline Benzene $2-[(1,1-$ Dimethylethyl)sulfonyl] -2 -methyl- & 3 & 0.005 & 178 & 1 & S-111 \\
\hline Ethylsulfonyl- & 1 & 0.088 & 170 & 12 & $C-109$ \\
\hline $\begin{array}{l}\text { THIOCARBOXYLIC ACIDS, ESTERS AND AMID } \\
\text { Butanethioic Acid Ester }\end{array}$ & & & & & \\
\hline $\begin{array}{l}\text { S-Decyl Ester } \\
\text { Benzenesulfonamide }\end{array}$ & 3 & 0.240 & 260 & 21 & U-106 \\
\hline N-Butyl- & 40 & 1.500 & 213 & 158 & BY-108 \\
\hline Total & 48 & 1.864 & & 197 & \\
\hline
\end{tabular}


Table 16b. Sulfur Compounds Observed in One Tank at a Concentration Less Than $0.025 \mathrm{mg} / \mathrm{m}^{3}$

\begin{tabular}{|c|c|c|c|c|c|c|}
\hline Compound & $\begin{array}{c}\text { Total Number } \\
\text { of } \\
\text { Observations }\end{array}$ & $\begin{array}{c}\text { Maximum } \\
\text { Concentration, } \\
\mathrm{mg} / \mathrm{m}^{3} \\
\end{array}$ & MW & $\begin{array}{c}\text { Maximum } \\
\text { Concentration, } \\
\text { ppbv } \\
\end{array}$ & $\begin{array}{c}\text { Tank with } \\
\text { Maximum } \\
\text { Concentration }\end{array}$ & $\begin{array}{l}\text { TWINS2 } \\
\text { (CAS) } \\
\text { Number }\end{array}$ \\
\hline Thiophene & & & & & & \\
\hline $\begin{array}{l}\text { 2-Methoxy-5-methyl- } \\
\text { Benzenesulfonamide }\end{array}$ & 1 & 0.023 & 128 & 4 & C-104 & $31053-55-1$ \\
\hline N-Ethyl-N-methyl- & 1 & 0.001 & 199 & 0.1 & BY -112 & $80-39-7$ \\
\hline N-Hexyl- & 1 & 0.005 & 241 & 0.4 & $C-108$ & $7250-80-8$ \\
\hline
\end{tabular}


Table 17a. Silicon Compounds

Compound

SILANES AND SILOXANES

Silane

Trimethyl-

Chlorotrimethyl-

Dimethoxydimethyl-

Silanol

Trimethyi-

Siloxane

Cyclotrisiloxane

Tetramethyl-

Hexamethyl-

Cyclotetrasiloxane

Octamethyl-

Decamethyl-

SILANE ESTERS

Ethanedioic Acid Ester

bis(TMS) Ester

2-[(TMSjoxy]benzoic Acid TMS Ester

alpha,4-bis(TMSoxy)benzeneacetic Acid Methyl Ester

TMS Ester

alpha-[(TMS)oxy]benzenepropanoic Acid Ester TMS Ester

(*)

(*)

(*)

(*)

(*)

(*)

Total Number

of

Observations

Maximum

Concentration, $\mathrm{mg} / \mathrm{m}^{3}$

MW

Maximum Concentration, ppbv

$0.044 \quad 74$

0.048

0.056

0.035

1.300

4.000

6.300

5.400

0.037

0.140

234

0.220

270

0.034

0.007

0.028

310

(*)

1-[4-((TMS)oxy)phenyl]-

Benzeneethanamine

$\mathrm{N}$-\{Perfluorophenyl]-beta,4-bis[TMSoxy]

Butanamide

Perfluoro-N-[2-TMSoxy-2-[4-TMSoxy]phenyl]ethyl-

(*)

(*)

Total

Note

$\left.{ }^{*}\right)$ The trimethylsilyl fragment is designated as TMS in this table
1

1

164
0.039

222

0.082

449

0.180

493

17.950
Tank with

Maximum

Concentration

TX-111

AX-102

S-102

A-103

A-103

A-103

C-103

C-103

A-103

A-102

A-103

BY-103

C- 107

A-102

S-102

U-112

$U-106$ 
Table 17b. Silicon Compounds Observed in One Tank at a Concentration Less Than $0.025 \mathrm{mg} / \mathrm{m}^{3}$

Compound

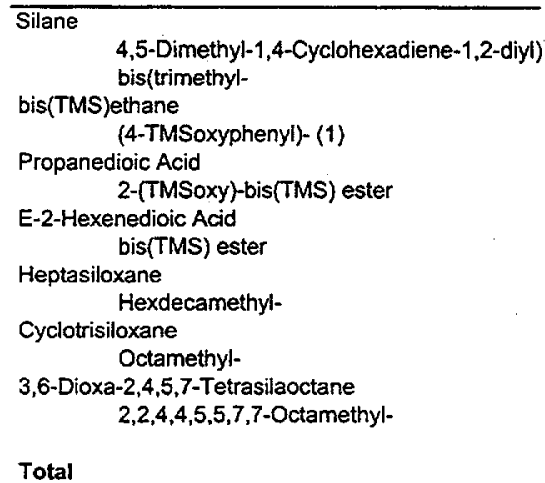

Total Number

of

Observations

1

1

1

1

1

1

1

7

7

Maximum Concentration, $\mathrm{mg} / \mathrm{m}^{3}$

0.004

0.004

0.009

0.022

0.014

0.021

0.079
Maximum Concentration, ppbv

MW

-

252

370

336

260

548

252

294

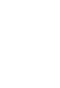

$\begin{array}{ll}0.079 & 2 \\ & 6\end{array}$

\begin{tabular}{rrr}
$\begin{array}{c}\text { Tank with } \\
\text { Maximum } \\
\text { Concentration }\end{array}$ & & $\begin{array}{c}\text { TWINS2 } \\
\text { (CAS) } \\
\text { Number }\end{array}$ \\
\cline { 3 - 3 } S-111 & & $101300-62-3$ \\
SX-103 & & US1000-04 \\
AX-102 & & $38165-93-4$ \\
SX-106 & & $55494-10-5$ \\
AX-102 & $541-01-5$ \\
SX-107 & US1000-09 \\
AX-102 & $4342-25-0$
\end{tabular}


Table 18a. Other Organic Compounds

\begin{tabular}{|c|c|c|c|c|c|}
\hline Compound & $\begin{array}{c}\text { Total Number } \\
\text { of } \\
\text { Observations }\end{array}$ & $\begin{array}{c}\text { Maximum } \\
\text { Concentration, } \\
\mathrm{mg} / \mathrm{m}^{3}\end{array}$ & MW & $\begin{array}{c}\text { Maximum } \\
\text { Concentration, } \\
\text { ppbv }\end{array}$ & $\begin{array}{c}\text { Tank with } \\
\text { Maximum } \\
\text { Concentration }\end{array}$ \\
\hline $\begin{array}{l}\text { ISOCYANATES } \\
\text { Methane }\end{array}$ & & & & & \\
\hline $\begin{array}{l}\text { isocyanato- } \\
\text { Propane }\end{array}$ & 2 & 0.046 & 57 & 18 & $U-106$ \\
\hline 2-Isocyanato- & 1 & 0.044 & 85 & 12 & $C-110$ \\
\hline $\begin{array}{l}\text { HYDRAZINES } \\
\text { Hydrazine }\end{array}$ & & & & & \\
\hline 1,1-Dimethyl- & 4 & 0.008 & 60 & 3 & $T X-105$ \\
\hline Total & 7 & 0.098 & & 33 & \\
\hline
\end{tabular}


Table 18b. Other Organic Compound Observed in One Tank at a Concentration Less Than $0.025 \mathrm{mg} / \mathrm{m}^{3}$

\begin{tabular}{|c|c|c|c|c|c|c|}
\hline Compound & $\begin{array}{c}\text { Total Number } \\
\text { of } \\
\text { Observations }\end{array}$ & $\begin{array}{c}\text { Maximum } \\
\text { Concentration, } \\
\mathrm{mg} / \mathrm{m}^{3}\end{array}$ & MW & $\begin{array}{c}\text { Maximum } \\
\text { Concentration, } \\
\text { ppbv }\end{array}$ & $\begin{array}{c}\text { Tank with } \\
\text { Maximum } \\
\text { Concentration }\end{array}$ & $\begin{array}{l}\text { TWINS2 } \\
\text { (CAS) } \\
\text { Number }\end{array}$ \\
\hline Methyl- & 1 & 0.0028 & 46 & 1 & TY-101 & $60-34$ \\
\hline
\end{tabular}


Table 19. Inorganic Compounds

\begin{tabular}{|c|c|c|c|c|c|c|}
\hline Compound & $\begin{array}{c}\text { Total Number } \\
\text { of } \\
\text { Observations }\end{array}$ & $\begin{array}{c}\text { Maximum } \\
\text { Concentration, } \\
\mathrm{mg} / \mathrm{m}^{3}\end{array}$ & MW & $\begin{array}{c}\text { Maximum } \\
\text { Concentration, } \\
\text { ppmv } \\
\end{array}$ & $\begin{array}{c}\text { Tank with } \\
\text { Maximum } \\
\text { Concentration } \\
\end{array}$ & $\begin{array}{l}\text { TWINS2 } \\
\text { (CAS) } \\
\text { Number }\end{array}$ \\
\hline Hydrogen & 98 & 115.45 & $\overline{2}$ & 1293.0 & C-107 & $1333-74-0$ \\
\hline Carbon Monoxide & 25 & 125.00 & 28 & 100.0 & A-106 & $630-08-0$ \\
\hline Carbon Dioxide & 101 & 3411.96 & 44 & 1737.0 & 8-102 & $124-38-9$ \\
\hline Nitrous Oxide & 123 & 2160.71 & 44 & 1100.0 & $U-102$ & $10024-97-2$ \\
\hline Nitric Oxide & 51 & 2.14 & 30 & 1.6 & $C-103$ & $10102-43-9$ \\
\hline Nitrogen Dioxide & 16 & 0.43 & 16 & 0.6 & $B Y-102$ & $10102-44-0$ \\
\hline Ammonia & 141 & 791.56 & 17 & 1043.0 & $B Y-108$ & $7664-41-7$ \\
\hline Sulfur Oxides & 1 & & 60,84 & 0.4 & C-104 & UIN000-01 \\
\hline Carbonyl Sulfide & 2 & 0.07 & 60 & 0.03 & TY-101 & $463-58-1$ \\
\hline Carbon Disulfide & 5 & 2.70 & 76 & 0.8 & C-103 & $75-15-0$ \\
\hline Total & 563 & 6610 & & 5276 & & \\
\hline
\end{tabular}


Table 20. Tanks with Highest Organic Carbon Content in Dome Space Determined by Total Non Methane Hydrocarbon Content Measurements, GC-MS Analysis of Summa Canisters or TST Samples (PNNL 1999)

Based on TNMHC

\begin{tabular}{lr}
\hline \multicolumn{1}{c}{ Tank } & $\begin{array}{c}\text { Concentration, } \\
\mathbf{m g} / \mathbf{m} 3\end{array}$ \\
C-103 & 520 \\
BY-108 & 310 \\
C-204 & 170 \\
BX-104 & 130 \\
BY-101 & 57 \\
BX-103 & 52 \\
TY-103 & 51 \\
S-102 & 28 \\
BY-102 & 20 \\
TX-111 & 16
\end{tabular}

Based on SUMMA

\begin{tabular}{rr}
\hline \multicolumn{1}{c}{ Tank } & \multicolumn{2}{c}{$\begin{array}{c}\text { Concentration, } \\
\mathbf{m g} / \mathbf{m} 3\end{array}$} \\
BY-104 & 210 \\
BY-108 & 170 \\
C-102 & 130 \\
C-101 & 87 \\
BY-107 & 84 \\
BX-103 & 55 \\
BY-110 & 50 \\
BX-104 & 49 \\
BY-101 & 47 \\
TY-103 & 43
\end{tabular}

Based on TST

\begin{tabular}{rr}
\hline \multicolumn{1}{c}{ Tank } & $\begin{array}{c}\text { Concentration, } \\
\mathrm{mg} / \mathrm{m} 3\end{array}$ \\
BY-103 & 2100 \\
$\mathrm{C}-108$ & 590 \\
$\mathrm{BY}-102$ & 290 \\
$\mathrm{BY}-108$ & 170 \\
$\mathrm{C}-101$ & 100 \\
BX-104 & 98 \\
TY-103 & 85 \\
BY-101 & 65 \\
BY-104 & 48 \\
& 45
\end{tabular}


Table 21. Summary of Observations

Category

Alkanes

Cycloalkanes

Alkenes and Alkadienes

Alkynes

Arenes

Halogen Compounds

Alcohols, Phenols and Ethers

Aldehydes

Ketones

Acids

Esters

Nitriles

Amines and Amides

Nitro and Nitroso Compounds

Heterocycles

Sulfur Compounds

Silicon Compounds

Miscellaneous Organic Compounds

is

Inorganic Compounds

Total
Total Number of

less than

$0.025 \mathrm{mg} / \mathrm{m}^{3}$

of Different

Compounds

200

46

41

40
2

2
22

22

56

18

45

31

5
18

10
51

51

13

2
0

431
Total Number of

Observed Only

Once

Sum of Maximum

Concentration of

Total Number of Compounds

at more than Compounds at more than $\mathbf{5 0 0}$ ppbv

Total Number of Compounds at

Compounds, ppmv $\quad 1000$ ppbv

ppb 


\section{Distribution}

No. of

Copies

OFFSITE

L. M. Stock

6695 SW 86th Avenue

Portland, OR 97223

\section{ONSITE}

16 CH2M HILL and Affiliated Companies

J. C. van Keuren

B4-47

N. K. Butler

R3-25

R. J. Cash

W. L. Cowley (3)

J. S. Davis

J. E. Meacham

D. A. Reynolds (5)

K. R. Sandgren

A. E. Young

Central Files
R1-44

R1-44

R1-49

R1-49

R2-11

R1-49

R1-10

B1-07
No. of

\section{Copies}

15 Pacific Northwest National Laboratory

J. W. Brothers (3)

K9-20

J. L. Huckaby (5)

K7-15

Information Release Office (7)

Distr.1 